\title{
Effect of End Friction on the Dynamic Compressive Mechanical Behavior of Concrete under Medium and Low Strain Rates
}

\author{
Liu Jin, ${ }^{1,2}$ Chengshun Xu, ${ }^{1}$ Yaqiang Han, ${ }^{1}$ and Xiuli Du ${ }^{1}$ \\ ${ }^{1}$ Key Laboratory of Urban Security and Disaster Engineering, Ministry of Education, Beijing University of Technology, \\ Beijing 100124, China \\ ${ }^{2}$ Department of Civil Engineering, Tsinghua University, Beijing 100084, China
}

Correspondence should be addressed to Chengshun Xu; xuchengshun@bjut.edu.cn

Received 8 September 2015; Revised 25 October 2015; Accepted 26 October 2015

Academic Editor: Nerio Tullini

Copyright (C) 2016 Liu Jin et al. This is an open access article distributed under the Creative Commons Attribution License, which permits unrestricted use, distribution, and reproduction in any medium, provided the original work is properly cited.

\begin{abstract}
The objective of the study is to examine the quantitative influence of end friction on the dynamic mechanical behavior of concrete under medium and low strain rates. Considering the concrete heterogeneity, a mesoscale mechanical model was established to study the confinement effect of end friction, in which the concrete was assumed to be composed of aggregates, mortar matrix, and the interfacial transition zones between them. The friction behavior was utilized to describe the interaction between the concrete specimens and loading apparatus. The dynamic axial compressive mechanical behavior of concrete subjected to different medium and low strain rates and friction coefficients was simulated. Furthermore, the confinement mechanism of end friction on the compressive dynamic increase factor (DIF) of concrete was studied. The simulation results indicate that with the increase of end friction coefficient, the uniaxial compressive strength of concrete first increases and then becomes stable; the end friction confinement changes the local stress state and damage distribution of concrete, and it thus contributes to the increase in compressive strength of concrete; the friction contribution factor presents a descending tendency with increasing the strain rate and decreases obviously when the end friction coefficient increases.
\end{abstract}

\section{Introduction}

Strain rate effects on the tensile and compressive strengths of concrete-like materials play an important role in the construction of material constitutive models which, in turn, exert a great deal of influences on the numerical simulations of concrete structures subjected to intense dynamic loadings [1]. The dynamic increase factor [DIF], defined by the ratio of the dynamic strength to the quasistatic strength in uniaxial compression and tension, has been widely accepted as an important parameter to measure the strain-rate effect on the strength of concrete-like materials [2]. A great number of tests have been carried out to find the dependence of DIF on strain-rate by using various test methods, for example, drophammer techniques, servo-hydraulic loading rigs, Split Hopkinson pressure bar [SHPB], and explosive devices [2-4]. The obtained test data demonstrates the strain rate sensitivity of concrete dynamic strengths and indicates that the tested DIF increases with strain rate. However, the test data presents large discrepancy, which may come from the following sources: (1) different testing techniques; (2) specimen size effect; (3) materials differences, for example, concrete quality, aggregate grade, curing and moisture condition, and age, and (4) dynamic and boundary effects as well as specimen lateral inertial effect.

Recently, many efforts have been conducted to explore the mechanism of apparent dynamic strength enhancement of concrete, such as in the publications $[1,2]$. It is widely agreed now that the DIF obtained from dynamic test is affected by lateral inertia confinement $[4,5]$. The friction at the interface of concrete specimen and loading measurement in the testes may also constrain the lateral deformation of the specimen and changes the internal stress status, and thus it might affect the test results as well. The strain rate effect described by the tensile and compressive DIFs should purely reflect the strainrate-dependence of material itself. 
As known, the end friction cannot be completely avoided when conducting dynamic compressive tests. The quantitative influence of end friction confinement should be deducted in the compressive DIF and it has been an interesting topic. To discover the influence of end friction on the macroscopic mechanical behavior of concrete, including the dynamic failure patterns and the nominal stress-strain curves as well as the compressive DIF of concrete subjected to dynamic loadings, numerous efforts have been conducted. For instance, using the numerical methods, Li and Meng [2] discussed the end friction confinement effect and found that, for specimens with $L / D=0.5$ [ $L$ means length of specimen and $D$ means the diameter of the specimen], the influence of the friction coefficient $\mu$ on DIF is insignificant when $\mu$ is less than 0.1 but becomes considerable when it is larger than 0.2. In their simulations, the strain rate employed ranges from 2.7 $\times 10^{1} \mathrm{~s}^{-1}$ to $3.9 \times 10^{2} \mathrm{~s}^{-1}$, and the friction coefficient utilized ranges from 0 to 0.7 . $\mathrm{Mu}$ et al. [6] modeled the dynamic mechanical properties of concrete subjected to the strain rate ranging from $4.4 \times 10^{1} \mathrm{~s}^{-1}$ to $1.47 \times 10^{2} \mathrm{~s}^{-1}$. They found that the compressive strain-rate effect of concrete-like materials is a pseudo property, and the main mechanisms that initiate lateral confinement include the material lateral inertia and the interface friction between the loading apparatus and the loaded specimen. In addition, in the works of Kim et al. [7], Li et al. [40], and Hao et al. [4], the interface friction between the loading apparatus and the loaded specimen was also simulated and examined. They concluded that the friction effect is another factor in the so-called strain-rate effect. These simulation efforts contribute to the understandings about the dynamic compressive strength enhancement of concrete. However, these efforts are all focused on high strain rates, and the effect of end friction on the failure of concrete under medium and low strain rates is rarely involved.

The objective of the present study is, therefore, to examine the quantitative influence of the confinement due to end friction on the mechanical behavior of concrete under medium and low strain rates. Experimental results show that concrete is a heterogeneous brittle material that fractures through the formation, growth, and coalescence of microcracks [8]. Moreover, the failure behavior of concrete is closely related to the meso-/microstructure of concrete, and the heterogeneities should therefore be considered [9-13]. In this work, concrete was assumed to be a composite material consisting of mortar matrix, coarse aggregates, and interfacial transition zone (ITZ) between the former two phases. Including these components in both experimental and numerical investigations is essential because the mechanical properties of these materials are distinct. In the computational model, the damaged plasticity model implemented in a strain-rate sensitive framework was utilized for the description of the dynamic mechanical behavior of the mortar matrix and the ITZ, and elastic model was employed to characterize the mechanical properties of the aggregate. The friction behavior was utilized to describe the interaction between the concrete specimens and loading apparatus. By using the material model, together with the commercial software ABAQUS, the reliability of the present approach was verified through the comparison with the available test data. Subsequently, the dynamic axial compressive mechanical behavior of concrete subjected to different medium and low strain rates was simulated under different friction coefficients. Furthermore, the confinement mechanism of end friction on the compressive dynamic increase factor (DIF) of concrete was studied and discussed.

The paper is decomposed as follows. The next section describes the rate-dependent constitutive framework for concrete. Section 3 presents the mesoscale computational model for the simulation of the end friction confinement effect and illustrates the verification of the present approach. In Section 4, the quantitative influences of the end friction confinement on the failure patterns, the nominal stress-strain curves, and the compressive DIF of concrete are explored. And finally, the concluding remarks are given in Section 5.

\section{The Constitutive Framework for Concrete Mesocomponents}

2.1. Concrete Damaged Plasticity Model. The behavior of concrete is complex due to an array of morphological features as well as deformation and failure mechanisms inherent in the concrete microstructure. The complicating factors include heterogeneous constitution, random phase distribution, arbitrary microcrack patterns, and internal granular friction. The complexity of the meso- or microscopic processes renders explicit resolution of the influences on a highly challenging task.

As known, plasticity, continuum damage mechanics, fracture mechanics, elastic-damage, and combined plasticity and damage mechanics are the common theories that have been widely used for the description of concrete mechanical behavior. However, the plasticity theory failed to address the degradation of the material stiffness due to microcracking, the fracture mechanics method was hard to reflect the nucleation of numerous microcracks and the growth of the microcracks to macrocracks, and the continuum damage mechanics with elasticity also could not describe the irreversible deformations and the inelastic volumetric expansion in compression [13]. Since both the stiffness degradation behavior and irreversible plastic deformations are contributing to the nonlinear behavior of concrete, a reasonable constitutive model should characterize the two distinct mechanisms. In recent years, many coupled plasticity-damage models were proposed to describe the mechanical behavior of concrete, such as the work in the references [13, 15-18]. Among these efforts, the concrete damaged plasticity model was first proposed by Lubliner et al. [15] for monotonic loading, and it was developed later by Lee and Fenves [16] to consider the dynamic and cyclic loadings. The model is a continuum, plasticity based model for concrete and it assumes that the main failure mechanisms of the concrete are cracking in tension and crushing in compression. The model which has been included in the ABAQUS package has been widely used for the description of the static and dynamic mechanical behaviors of concretelike materials, for example, in the publications [11, 12, 19, 20]. A brief presentation of the damaged plasticity model from ABAQUS is presented below. 


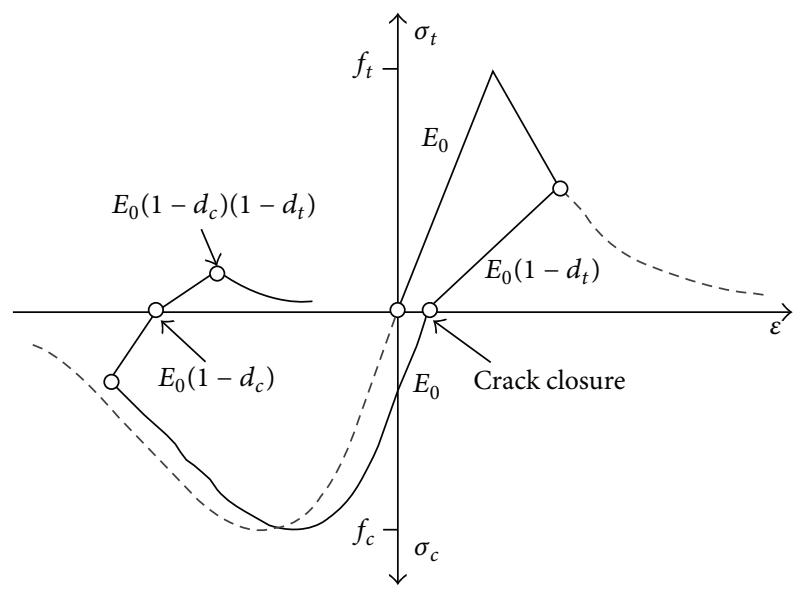

FIGURE 1: Unaxial loading path of concrete plasticity damage model.

2.1.1. Isotropic Damage Model. The stress-strain relation for the general three-dimensional multiaxial condition is given by the scalar damage elasticity equation:

$$
\sigma=(1-d) D_{0}^{\mathrm{el}}:\left(\varepsilon-\varepsilon^{\mathrm{pl}}\right)
$$

in which $D_{0}^{\mathrm{el}}$ is the initial elasticity matrix, $d$ means the damage variable, and $\varepsilon^{\mathrm{pl}}$ is the plastic strain tensor. Tensile damage and compressive damage are characterized independently by two hardening parameters, $\widetilde{\varepsilon}_{t}^{\mathrm{pl}}$ and $\widetilde{\varepsilon}_{c}^{\mathrm{pl}}$ (subscripts " $t$ " and " $c$ " stand for tension and compression, resp.), which are referred to as equivalent plastic strains in tension and compression. The evolution equations of the hardening variables, $\widetilde{\varepsilon}_{t}^{\mathrm{pl}}$ and $\widetilde{\varepsilon}_{c}^{p l}$, are conveniently formulated by considering uniaxial loading conditions first and then extended to multiaxial conditions. The model assumes that the uniaxial tensile and compressive responses of concrete are characterized by damaged plasticity, as shown in Figure 1. It is assumed that the stressstrain response follows a linear elastic relationship under uniaxial tension until the value of the failure stress, $f_{t}$, is reached. The failure stress corresponds to the onset of microcracking in the concrete material. Beyond the failure stress the formation of microcracks is often represented macroscopically with a softening stress-strain response, which induces strain localization in the concrete structure. The uniaxial stressstrain curves can be converted into stress versus plastic strain curves of the form as

$$
\begin{aligned}
& \sigma_{t}=\sigma_{t}\left(\widetilde{\varepsilon}_{t}^{\mathrm{pl}}, \dot{\vec{\varepsilon}}_{t}^{\mathrm{pl}}, \theta, f_{i}\right), \\
& \sigma_{c}=\sigma_{c}\left(\widetilde{\varepsilon}_{c}^{\mathrm{pl}}, \dot{\bar{\varepsilon}}_{c}^{\mathrm{pl}}, \theta, f_{i}\right),
\end{aligned}
$$

where $\dot{\bar{\varepsilon}}_{t}^{\mathrm{pl}}$ and $\dot{\bar{\varepsilon}}_{c}^{\mathrm{pl}}$ are the equivalent plastic strain rates; $\theta$ is the temperature; and $f_{i}(i=1,2, \ldots)$ are other predefined field variables.

As shown in Figure 1, when the specimen is unloaded from any point on the strain softening branch of the stressstrain curves, the unloading response is weakened: the elastic stiffness of the material appears to be degraded. Herein, the degradation of the elastic stiffness is characterized by two damage variables, tensile damage factor $d_{t}$ and compressive damage factor $d_{c}$, and the stress-strain relationships under tension and compression are, respectively,

$$
\begin{aligned}
& \sigma_{t}=\left(1-d_{t}\right) E_{0}\left(\varepsilon_{t}-\widetilde{\varepsilon}_{t}^{\mathrm{pl}}\right), \\
& \sigma_{c}=\left(1-d_{c}\right) E_{0}\left(\varepsilon_{c}-\widetilde{\varepsilon}_{c}^{\mathrm{pl}}\right) .
\end{aligned}
$$

This representation, although somewhat simplified, captures the main features of concrete behavior. Sometimes, in order to avoid unreasonable mesh sensitive results, the tensile post-failure behavior is given in terms of a fracture energy cracking criterion by specifying a stress-displacement curve instead of a stress-strain curve. Under multiaxial loading conditions, the evolution equation of the hardening parameters for uniaxial loading should be extended.

According to Lee and Fenves's work [16], tensile and compressive equivalent plastic strains have the following forms:

$$
\begin{aligned}
& \widetilde{\varepsilon}_{t}^{\mathrm{pl}}=r(\widehat{\bar{\sigma}}) \widehat{\varepsilon}_{\max }^{\mathrm{pl}}, \\
& \widetilde{\varepsilon}_{c}^{\mathrm{pl}}=-(1-r(\widehat{\bar{\sigma}})) \widehat{\varepsilon}_{\text {min }}^{\mathrm{pl}}
\end{aligned}
$$

in which $\widehat{\varepsilon}_{\max }^{\mathrm{pl}}$ and $\widehat{\varepsilon}_{\min }^{\mathrm{pl}}$ are the maximum and minimum plastic strain tensors, respectively; $r(\hat{\bar{\sigma}})$ means a multiaxial stress weight factor and is defined as

$$
r(\hat{\bar{\sigma}})=\frac{\sum_{i=1}^{3}\left\langle\hat{\bar{\sigma}}_{i}\right\rangle}{\sum_{i=1}^{3}\left|\hat{\bar{\sigma}}_{i}\right|} \quad 0 \leq r(\hat{\bar{\sigma}}) \leq 1
$$

in which $\hat{\bar{\sigma}}_{i}$ are principal stress components $(i=1,2$ for $2 \mathrm{D}$ problems and $i=1,2,3$ for 3D problems) and the Macauley bracket " $\langle\cdot\rangle$ " is defined by $\langle x\rangle=0.5 \times(|x|+x)$.

2.1.2. Plasticity Yield Surface. The model makes use of the yield function of Lubliner et al. [15], with the modifications proposed by Lee and Fenves [16] to account for different evolution of strength under tension and compression. The evolution of the yield surface is controlled by the two hardening variables, $\widetilde{\varepsilon}_{t}^{\mathrm{pl}}$ and $\widetilde{\varepsilon}_{c}^{\mathrm{pl}}$. In terms of effective stress, the yield function $F$ is defined as

$$
\begin{aligned}
F= & F\left(\bar{\sigma}, \widetilde{\varepsilon}^{\mathrm{pl}}\right) \\
= & \frac{1}{1-\alpha}\left[\bar{q}-3 \alpha \bar{p}+\beta\left(\widetilde{\varepsilon}^{\mathrm{pl}}\right)\left\langle\bar{\sigma}_{\max }\right\rangle-\gamma\left\langle-\bar{\sigma}_{\max }\right\rangle\right] \\
& -\bar{\sigma}_{c}\left(\varepsilon_{c}^{\mathrm{pl}}\right)=0
\end{aligned}
$$

in which $\bar{p}=-(1 / 3) \bar{\sigma}: I$ is the effective hydrostatic pressure stress; $I$ is a stress invariant; $\bar{q}=\sqrt{(3 / 2) \bar{S}: \bar{S}}$ means the Mises equivalent effective stress; and $\bar{S}$ is the effective stress deviator, defined as $\bar{S}=\bar{p} I+\bar{\sigma} ; \bar{\sigma}_{\max }$ is the maximum principal effective stress. Typical yield surface is depicted in Figure 2 for plane stress conditions. 


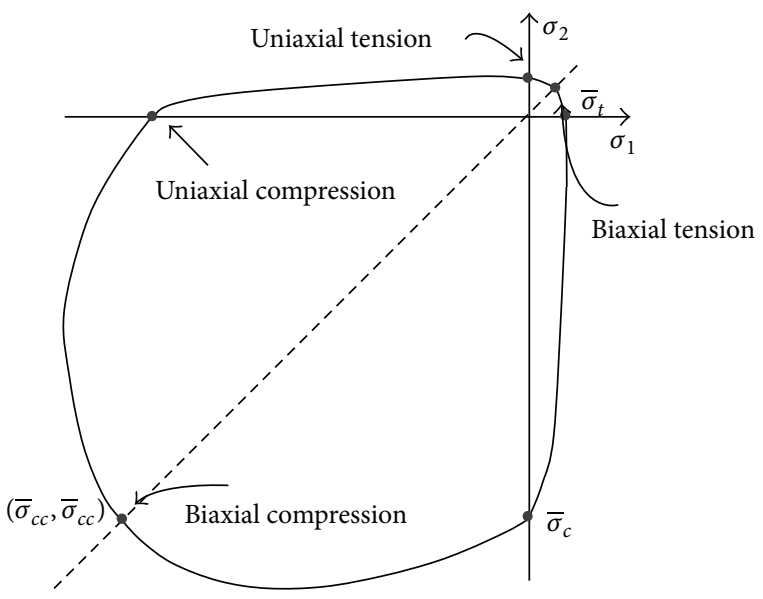

FIGURE 2: Yield surface for plane stress conditions.

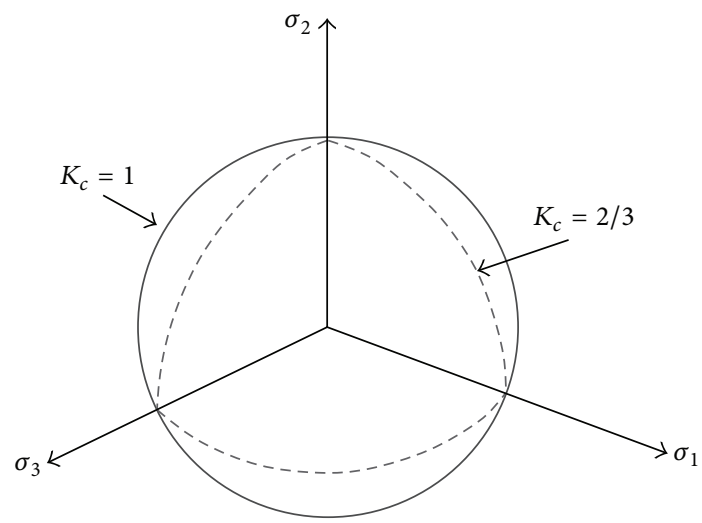

FIGURE 3: Yield surfaces in the deviatory plane $\left[K_{c}=2 / 3\right.$ corresponds to Rankine formulation and $K_{c}=1$ corresponds to the Drucker-Prager criterion].

Moreover, parameters of $\alpha, \beta\left(\widetilde{\varepsilon}^{\mathrm{pl}}\right)$, and $\gamma$, respectively, can be calculated by

$$
\begin{aligned}
\alpha & =\frac{\bar{\sigma}_{c c} / \bar{\sigma}_{c}-1}{2 \bar{\sigma}_{c c} / \bar{\sigma}_{c}-1}, \\
\beta\left(\widetilde{\varepsilon}^{\mathrm{pl}}\right) & =\frac{\bar{\sigma}_{c}\left(\widetilde{\varepsilon}_{c}^{\mathrm{pl}}\right)}{\bar{\sigma}_{t}\left(\widetilde{\varepsilon}_{t}^{\mathrm{pl}}\right)}(1-\alpha)-(1+\alpha), \\
\gamma & =\frac{3\left(1-K_{c}\right)}{2 K_{c}-1}
\end{aligned}
$$

in which $\bar{\sigma}_{c c} / \bar{\sigma}_{c}$ means the ratio of initial equivalent-biaxial compressive yield stress to initial uniaxial compressive yield stress and its default value of the ratio is 1.16 according to $[19,20] ; K_{c}$ is the ratio of the tensile to the compressive meridian and defines the shape of the yield surface in the deviatory plane (see Figure 3).

2.1.3. Plastic Flow Rule. The nonassociated potential plastic flow is employed in the present damaged plasticity model. The flow potential function $G(\sigma)$ used for this model is the nonassociated Drucker-Prager hyperbolic function and is defined according to

$$
G(\sigma)=\sqrt{\left(\eta \sigma_{t} \tan \psi\right)^{2}+\bar{q}^{2}}-\bar{p} \tan \psi
$$

in which $\psi$ is the dilation angle measured in the $p$ - $q$ plane at high confining pressure; $\sigma_{t}$ is the uniaxial tensile stress at failure; $\eta$ is the eccentricity that gives the rate at which the plastic potential function approximates the asymptote (the flow potential tends to a straight line as the eccentricity tends to zero); and its default value is equal to 0.1 . The nonassociated flow rule means that the plastic strain vector is normal to the plastic potential function that differs from the yield surface.

The plastic strain rate $\dot{\varepsilon}^{\mathrm{pl}}$ and the effective plastic strain rate $\dot{\bar{\varepsilon}}^{\mathrm{pl}}$ can be derived as follows:

$$
\begin{aligned}
& \dot{\mathcal{\varepsilon}}^{\mathrm{pl}}=\dot{\lambda} \frac{\partial G}{\partial \sigma} \\
& \dot{\dot{\mathcal{\varepsilon}}}^{\mathrm{pl}}=h\left(\sigma, \widetilde{\mathcal{\varepsilon}}^{\mathrm{pl}}\right) \cdot \dot{\varepsilon}^{\mathrm{pl}}
\end{aligned}
$$

where $\dot{\lambda}$ is the plastic multiplier.

Because the selected plastic flow is nonassociated, to obtain an acceptable rate of convergence, the unsymmetric matrix storage and solution scheme is used. For more details about the constitutive model refer to Lubliner et al. [15], Lee and Fenves [16], and Du et al. [11].

2.2. Dynamic Increase Factor (DIF) for Concrete. The available experimental and numerical observations have demonstrated that concrete and other cementitious materials are rate sensitive $[3,21,22]$. The strain rate effect has been studied on different mechanical characteristics of concrete: Young's modulus, Poisson's ratio, energy-absorption capacity, and axial strain at maximum strength are rate-sensitive quantities, but at a much lower intensity than the compressive and tensile strengths $[3,23]$. Similar to the work $[4,11]$, only the strength enhancement was considered in the present study.

So far, many empirical and theoretical relations have been developed to quantitatively describe the strain rate effect on concrete properties. In particular, the code by the Comite Euro-international du Beton (CEB) recommends various values of the DIF for the tensile and compressive strengths under different strain rates based on the available test results. The formula of the compressive DIF recommended by CEB [3] has the following form:

$$
\begin{aligned}
& \mathrm{CDIF}=\frac{f_{\mathrm{cd}}}{f_{\mathrm{cs}}}=\left(\frac{\dot{\varepsilon}_{d}}{\dot{\varepsilon}_{\mathrm{cs}}}\right)^{1.026 \alpha} \quad \text { for } \dot{\varepsilon}_{d} \leq 30 \mathrm{~s}^{-1} \\
& \mathrm{CDIF}=\frac{f_{\mathrm{cd}}}{f_{\mathrm{cs}}}=\gamma\left(\dot{\varepsilon}_{d}\right)^{1 / 3} \quad \text { for } \dot{\varepsilon}_{d}>30 \mathrm{~s}^{-1},
\end{aligned}
$$

where $f_{\mathrm{cd}}$ is the dynamic compressive strength at the strain rate of $\dot{\varepsilon}_{d}$ (in the range of $30 \times 10^{-6}-1000 \mathrm{~s}^{-1}$ ), $\dot{\varepsilon}_{\mathrm{cs}}=30 \times$ $10^{-6} \mathrm{~s}^{-1}$ (static strain rate), $\log \gamma=6.156 \alpha-0.49, \alpha=(5+$ $\left.3 f_{\mathrm{cu}} / 4\right)^{-1}, f_{\mathrm{cs}}$ is the static compressive strength, and $f_{\mathrm{cu}}$ is the static cube compressive strength [in MPa]. The DIF for 
the compressive strength in (10a) and (10b) was often adopted, for example, in the publications [10, 11].

The CEB also gives empirical formulae to estimate the DIF for concrete tensile strength. However, comparison between the available experimental results in the literature with the $\mathrm{CEB}$ empirical equations indicates that the $\mathrm{CEB}$ tensile DIF substantially underestimates the tensile DIF of concrete [24, 25]. In Malvar and Ross's [24] work, an empirical formula was proposed, and the formula for the tensile DIF can be written as

$$
\begin{aligned}
& \text { TDIF }=\frac{f_{\mathrm{td}}}{f_{\mathrm{ts}}}=\left(\frac{\dot{\varepsilon}_{d}}{\dot{\varepsilon}_{\mathrm{ts}}}\right)^{\delta} \quad \text { for } \dot{\varepsilon}_{d} \leq 1 \mathrm{~s}^{-1}, \\
& \mathrm{TDIF}=\frac{f_{\mathrm{td}}}{f_{\mathrm{ts}}}=\beta\left(\frac{\dot{\varepsilon}_{d}}{\dot{\varepsilon}_{\mathrm{ts}}}\right)^{1 / 3} \quad \text { for } \dot{\varepsilon}_{d}>1 \mathrm{~s}^{-1},
\end{aligned}
$$

where $f_{\text {td }}$ and $f_{\text {ts }}$ are, respectively, the dynamic uniaxial tensile strength at the strain rate $\dot{\varepsilon}_{d}$ and the quasistatic uniaxial tensile strength, $\dot{\varepsilon}_{\mathrm{ts}}=10^{-6} \mathrm{~s}^{-1}$ (static strain rate), $\log \beta=6 \delta-2$, $\delta=1\left(1+8 f_{c}^{\prime} / f_{c 0}^{\prime}\right), f_{c 0}^{\prime}=10 \mathrm{MPa}$, and $f_{c}^{\prime}$ is the quasistatic uniaxial compressive strength of concrete (in $\mathrm{MPa}$ ).

\section{Mesoscale Computational Model for Concrete}

Theoretical, experimental, and numerical studies indicate that the failure pattern, crack mode, and even the velocity of the crack propagation are influenced by loading rate $[11,26-$ 28]. The mechanical behaviors of concrete, including the macroscopic mechanical properties and the failure patterns, are associated with the micro-/mesostructure of concrete. Due to the complexity of concrete, the failure behavior can be studied numerically. In the numerical simulations, concrete heterogeneity should be accounted for, and thus it requires the adoption of a mesoscale mechanical model in which the heterogeneity can be explicitly described.

At the mesoscale, concrete was often assumed to be a multiphase composite composed of aggregate, mortar matrix, the interfacial transition zones [ITZs], voids, and so forth. A lot of work $[9,10,19,29]$ has demonstrated that the ITZs have a dramatic influence on the failure pattern and the macromechanical properties of concrete. However, the mechanical properties, especially the dynamic mechanical behavior of the ITZs, are not well understood yet [13, 19, 30-32]. Moreover, the real typical thickness of ITZ is about $10-50 \mu \mathrm{m}[9,10]$, which limits the minimum size of the finite element mesh. In most simulation efforts, the ITZ thickness is set as $0.2-0.8 \mathrm{~mm}$ $[9,10], 0.5 \mathrm{~mm}[8], 0.4 \mathrm{~mm}[19]$, and $1 \mathrm{~mm}[12]$ for the sake of simplicity.

3.1. Mesostructure and Mechanical Model of Concrete. Concrete exhibits heterogeneity in material and mechanical properties on many length scales [29]. At the mesoscale, considering the effect of heterogeneities of concrete, the concrete in the present work was regarded as a three-phase composite composed of coarse aggregate particles, mortar matrix, and the ITZs between the former two phases. Random aggregate models have long been demonstrated to be an effective mesomechanical approach to simulating the failure process and the deformation of concrete, and they have been widely utilized in fracture mechanics of concrete, for example, in [1113,33 . Based on the concrete random aggregate model in which the shape, size, and distribution of the coarse aggregate particles closely resemble real concrete in the statistical sense, lots of efforts have been carried out to investigate the macroscopic mechanical behavior and failure mechanism of concrete $[10-12,29]$. In this work, the concrete random aggregate model was therefore also employed.

Due to great computational expense and memory demand of mesoscale simulations, it is, in general, not possible to model the full structure at the mesoscale with the computational power available nowadays. Herein the present study, only two-dimensional simulations were thus conducted. Similar to the work [9-12,33], the aggregate particles are modeled as circles with random spatial distribution enfolded by a thin interface layer of ITZ. Similar to those in $[11,12]$, the "take-and-place" method was utilized to generate the coarse aggregate particles. The fine ones and other components (e.g., air voids) were assumed to be mixed up with the cement paste establishing the mortar matrix phase. This matrix phase can thus be regarded as a homogeneous component at the mesoscale. The material properties of the paste phase are thus strongly dependent on the type of cement used, amount of pores, water to cement ratio, and other additives present in the concrete, which can be determined by microscale simulation efforts. Furthermore, the Fuller curve widely utilized, for example, in [11, 12], was selected to determine the distribution of aggregate with different sizes employed in the concrete specimen. The aggregate particles were then placed into the matrix one by one at randomly determined positions and in such a way that no overlapping with particles already placed. Finally, at the mesoscale, aggregate particles (typically sand or gravel) and the surrounding ITZ layers behave as inclusions in the matrix phase of mortar matrix.

Figure 4 presents a typical schematic diagram of concrete mesostructure generated by employing the above approach. The size of the heterogeneous concrete specimen is $150 \mathrm{~mm} \times$ $150 \mathrm{~mm}$. Note that aggregate particles in real concrete are not perfectly circular. However, the efforts in $[10,11]$ have indicated that the effect of aggregate shape on macroscopic stress-strain curve of concrete seems to be very small. Similar to that in $[11,34]$, the concrete sample contains 6 medium stones with a diameter of $30 \mathrm{~mm}$ and 56 small stones with a diameter of $12 \mathrm{~mm}$. The concrete specimens involve about $46.9 \%$ of aggregate per volume. In Figure 4, the green areas mean the aggregate phase, the red thin-layer zones surrounding the aggregate particles are the ITZs, and the grey zones denote the mortar matrix. Herein, the interfacial zones are viewed as uniform thickness shells placed concentrically around each aggregate. Similar to the work [34], the thickness of the ITZ was set as $1 \mathrm{~mm}$.

To describe the influence of end friction confinement on the dynamic compressive mechanical properties of concrete, two rigid blocks (see the two blue blocks in Figure 4) were placed, respectively, at the top and the bottom of the concrete specimen. The interactions between the heterogeneous 

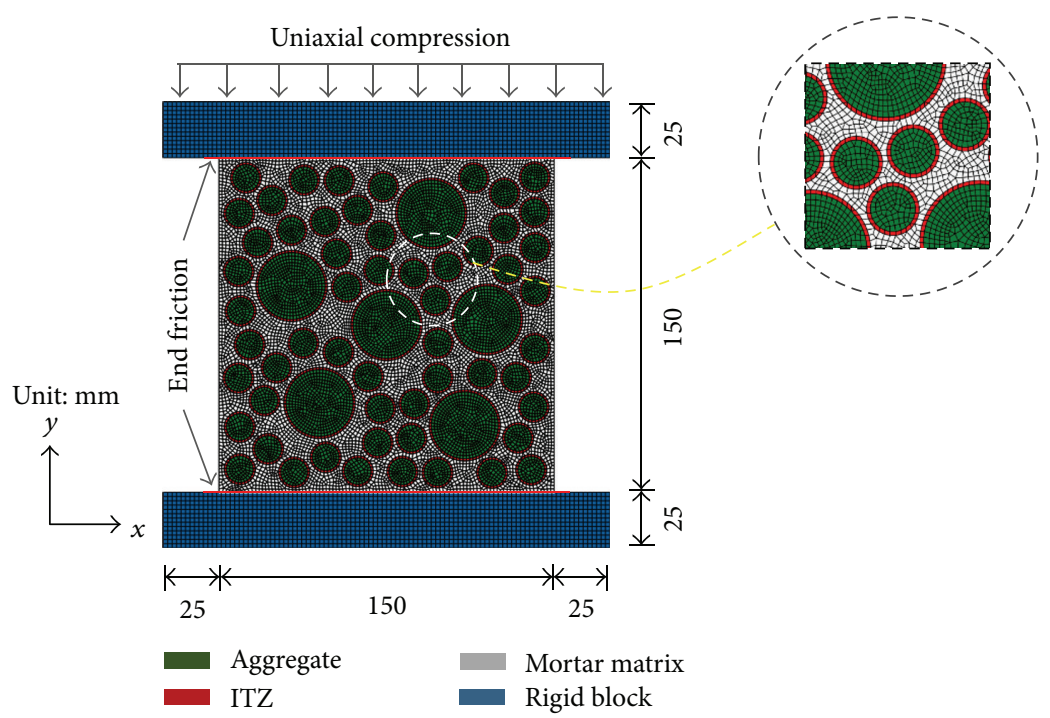

(1)

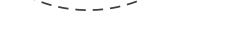

FiguRE 4: Mesoscale mechanical model of concrete under dynamic uniaxial compression, considering the influence of end friction confinement. The specimen has 22346 elements. [For interpretation of the references to color in this figure legend, the reader is referred to the web version of this paper].

concrete specimen and the two rigid blocks, that is, the two red-line regions, are implemented by the classic coulomb friction behavior. The friction coefficients considered in the following simulations are 0.0 (completely smooth), $0.1,0.2$, $0.3,0.6,0.8$, and $\infty$ (i.e., the top and bottom surfaces of the specimen were fixed in the horizontal direction) for a quantitative assessment of the end friction confinement effect. As known, the DIF difference obtained from the simulation results with different friction coefficients can then be attributed purely to the influence of end friction confinement effects.

The meshing of these concrete specimens were conducted with four node solid elements with a linear strain field. To have a better understanding of concrete fracture, the average mesh size was $1 \mathrm{~mm}$, which is consistent with that in the work from Hentz et al. [23] and Du et al. [11, 12].

3.2. Mechanical Parameters for the Three Mesocomponents. In Zhou and Hao's [11, 13] and Snozzi et al.s [35] work, the uniaxial tensile and compressive mechanical behavior of concrete at high strain rate were studied. From their simulation observations, it can be noticed that no damage generates in the aggregate phase. Moreover, in Pedersen et al.'s [8] simulation of the dynamic tensile behavior of concrete, the aggregate grains were assumed to follow a linear elastic material behavior. This is because the tensile and compressive strengths of the aggregate are much larger than those of mortar matrix and the ITZs. Similar to the work $[8,11]$, the aggregate was assumed as elastic in the following simulations. This treatment should work for the concrete subjected to medium and low strain rates, as discussed in the present study.

To the mortar matrix and the ITZs, the continuum damage mechanics theories mentioned previously were employed to describe their mechanical properties. In continuum mechanics, the constitutive model is often expressed in terms of stress-strain relations. When the material exhibits strain-softening behavior, leading to the strain localization behavior, this formulation leads to a strong mesh dependency of the finite element results in which the energy dissipated decreases upon mesh refinement $[11,19]$. Similar to the work in $[10,11,19]$, to avoid or release unreasonable mesh sensitive results, the tensile postfailure behavior was given in terms of a fracture energy cracking criterion by specifying a stressdisplacement curve instead of a stress-strain curve. In addition, unlike discrete crack models [36,37], the continuum damaged plasticity model based on continuum mechanics uses damage factors such as DAMAGEC for compression and DAMAGET for tension to describe the fracture behavior, and it is not capable of providing accurate crack geometry such as crack width.

The main mechanical parameters, including the elastic modulus, Poisson's ratio, and uniaxial tensile/compressive strengths of the three mesoconstituents used in this study for the following numerical analysis, were listed in Table 1.

It is to be noted that the DIFs given in Section 2.2 are built based on the test results on concrete material. The strain rate effects for the aggregate phase, mortar matrix, and the ITZ are essentially distinct, as illustrated in $[38,39]$. Nevertheless, Grote et al.s [21] experimental observations indicate that the dynamic mechanical behavior of mortar matrix is closely similar to that of concrete. In addition, the ITZ layer can be treated as a kind of mortar matrix with a high(er) porosity [34], and thus its dynamic mechanical behavior can also be regarded as similar to those of mortar matrix and concrete. In summary, it is assumed that the ITZ and the mortar matrix have the same DIFs mentioned previously. The treatments are consistent with the ones in $[9,10]$. The strain rate effect for both the mortar matrix and the ITZs on their tensile/compressive strengths was incorporated into the plasticity damaged model, which was represented by 
TABLE 1: Mechanical parameters of the concrete mesocomponents used in the study.

\begin{tabular}{lccc}
\hline & Mortar matrix & ITZ & Aggregate \\
\hline Mass density $\rho\left[\mathrm{kg} / \mathrm{m}^{3}\right]$ & $2.75 \times 10^{3}$ & $2.75 \times 10^{3}$ & $2.88 \times 10^{3}$ \\
Elastic modulus $[\mathrm{GPa}]$ & $30^{*}$ & $25^{*}$ & $70^{*}$ \\
Poisson's ratio & $0.2^{*}$ & $0.22^{*}$ & $0.16^{*}$ \\
Dilation angle $\psi\left[{ }^{\circ}\right]$ & 30 & 30 & \\
Eccentricity $\eta$ & 0.1 & 0.1 & \\
Shape factor $K_{c}$ & 0.667 & 0.667 & \\
Stress ratio $\bar{\sigma}_{c c} / \bar{\sigma}_{c}$ & 1.16 & 1.16 & \\
Tensile yield stress $\sigma_{t}[\mathrm{MPa}]$ & $1.43^{*}$ & $1.2^{*}$ & \\
Compressive yield stress $\sigma_{c 0}$ & $14.3^{*}$ & $12^{*}$ & \\
{$[\mathrm{MPa}]$} & & & \\
Ultimate compressive yield & 1.43 & 1.2 & \\
stress $\sigma_{\text {cu }}[\mathrm{MPa}]$ & & & \\
Note: data with “*” are quoted from Du et al. [12]. &
\end{tabular}

a dynamic increase factor (i.e., the DIF) with respect to strain rate.

3.3. Boundary/Loading Conditions and FE Simulations. To examine the effects of end friction confinement on the macromechanical behavior of concrete subjected to dynamic compressive loadings, the failure behavior of the concrete specimen was explored. The corresponding loading and boundary conditions for the interaction system were illustrated as follows: (1) constant vertical velocity $v$ was applied on the top surface of the rigid block, and the left and right sides of the specimen are free of stress; (2) the movement at the bottom rigid block was fixed at both the vertical and horizontal directions; (3) friction behavior between the two blocks and the concrete sample was set. The nominal strain rate $\dot{\varepsilon}$ is defined as the applied velocity divided by the height of the specimen; that is, $\dot{\varepsilon}=v / h$, where $h$ is the height of the specimen.

In the following simulations, ABAQUS increased automatically the size of step time going from smaller one to the maximum authorized, defined by the user. For all the concrete specimens, the numerical simulations were performed in a plane strain condition.

3.4. Verification of the Mesoscale Approach. In reality, the available tests on loading rate effect of concrete are not very rich, and the test data is also not complete. Comparatively speaking, Dilger et al.s [14] test data is the most complete one, and it is often used to verify the reliability of the analysis method or model, such as in [26].

To illustrate the reliability and accuracy of the present mesoscale simulation method, Dilger et al.s [14] test on the dynamic uniaxial compressive behavior of concrete was also selected for the comparison in this section. In Dilger et al's [14] experiment, the macroscopic mechanical behavior of concrete under different nominal strain rates (i.e., the strain rates of $3.33 \times 10^{-5} \mathrm{~s}^{-1}, 3.33 \times 10^{-3} \mathrm{~s}^{-1}$, and $0.2 \mathrm{~s}^{-1}$ ) was explored, and the complete compressive stress-strain curves of concrete were obtained.

In the previous work [33], a series of dynamic compressive test of concrete under different strain rates were conducted based on the present mesoscale mechanical method, including $\dot{\varepsilon}=3.33 \times 10^{-5} \mathrm{~s}^{-1}, 1 \times 10^{-4} \mathrm{~s}^{-1}, 3.33 \times 10^{-3} \mathrm{~s}^{-1}, 1 \times 10^{-2} \mathrm{~s}^{-1}$, $5 \times 10^{-2} \mathrm{~s}^{-1}, 0.2 \mathrm{~s}^{-1}, 1 \mathrm{~s}^{-1}, 100 \mathrm{~s}^{-1}$, and $200 \mathrm{~s}^{-1}$. It is to be noted that, in the simulations, the concrete specimens were fixed at one end and loaded longitudinally through an applied constant velocity at the other end. The detailed mechanical parameters and analysis method can refer to [33].

Figure 5 presents the failure patterns of the concrete specimen subjected to different nominal strain rates. One can notice that, with the increase of strain rate, the number of cracks (i.e., the red damaged region) increases obviously, and the crack branching behavior occurs. Therefore, with the increase of strain rate, the fracture of concrete requires more energy demand to reach failure, which should be the main reason for the improvement of the dynamic strength of concrete.

Figure 6(a) reports the comparison between the simulations results (solid curves) and the experimental data (symbols) in terms of nominal stress (applied forces divided by the specimen cross sectional area) versus nominal strain (the displacement generated at the applied-load end divided by the length of the specimen) curves, and the agreement is very good. It should be noted herein that, as mentioned previously, the related mechanical and physical parameters of the concrete mixture used in the test are not full, especially the mechanical parameters of the ITZ. Accordingly, in the previous work [33], the mechanical parameters (especially the elastic modulus and the strength) of the ITZ were determined by the inversion method on the basis of only two strain rates $\left(3.33 \times 10^{-5} \mathrm{~s}^{-1}\right.$ and $\left.3.33 \times 10^{-3} \mathrm{~s}^{-1}\right)$. The simulation of the third available strain (i.e., $0.2 \mathrm{~s}^{-1}$ ) can be regarded as a validation of the ability of the model to predict concrete strength outside the range of strain rates used for calibration. This treatment is similar to that in [26].

Furthermore, the compressive DIFs were evaluated according to the simulation results, and the comparison of the present simulation results and some available experimental observations is plotted in Figure 6(b). It can be observed that the present simulation results agree very well with the collective experimental data. This indicates that the present analysis method can well simulate the dynamic compressive behavior of concrete. The validated mesoscale mechanical approach would be employed in the following simulations to study the effect of end friction confinement on the dynamic compressive behavior of concrete.

\section{Dynamic Compressive Failure Behavior of Concrete}

On the basis of the mesoscale mechanical model described in Section 3, the simulations of the dynamic axial compression of the concrete specimen with various end friction coefficients were implemented. The loading velocities adopted on the upper rigid block in the simulations are 


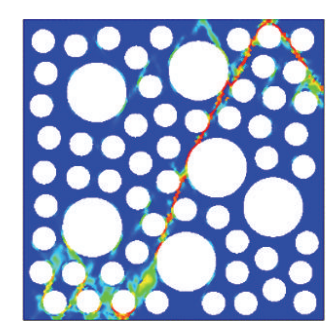

$\dot{\varepsilon}=3.33 \times 10^{-5}$

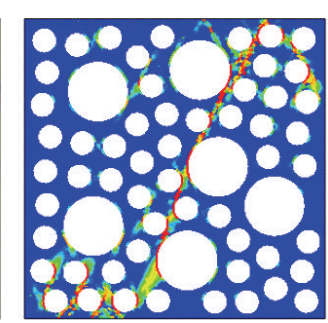

$\dot{\varepsilon}=1 \times 10^{-4}$

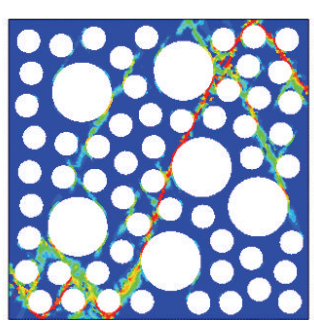

$\dot{\varepsilon}=3.33 \times 10^{-3}$

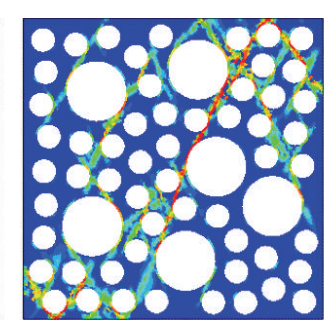

$\dot{\varepsilon}=1 \times 10^{-2}$

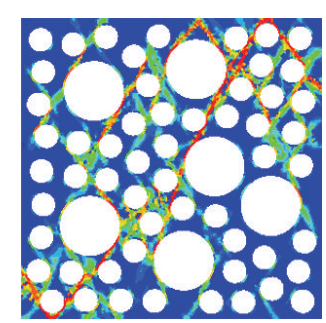

$\dot{\varepsilon}=5 \times 10^{-2}$

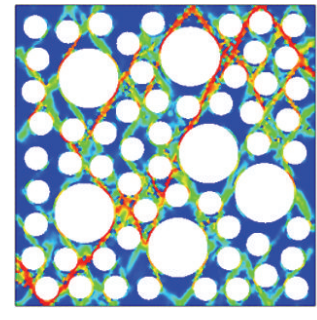

$\dot{\varepsilon}=2 \times 10^{-1}$

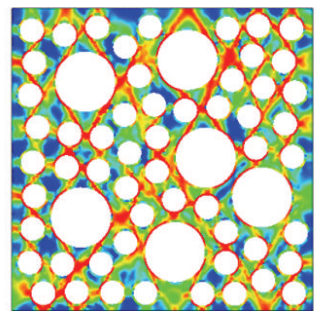

$\dot{\varepsilon}=1$

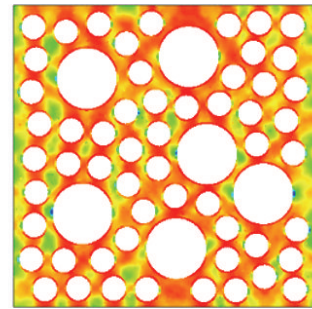

$\dot{\varepsilon}=100$

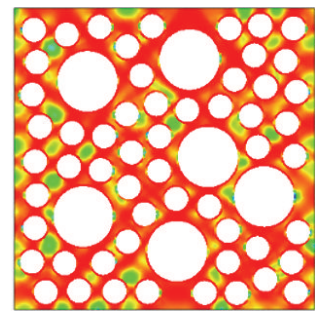

$\dot{\varepsilon}=200$

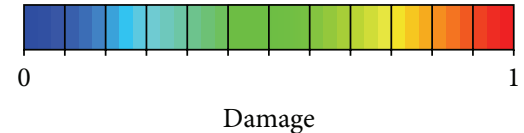

FIGURE 5: Compressive failure patterns of the concrete specimen under different strain rates ranging from $1 \times 10^{-5} \mathrm{~s}^{-1}$ to $200 \mathrm{~s}^{-1}$.

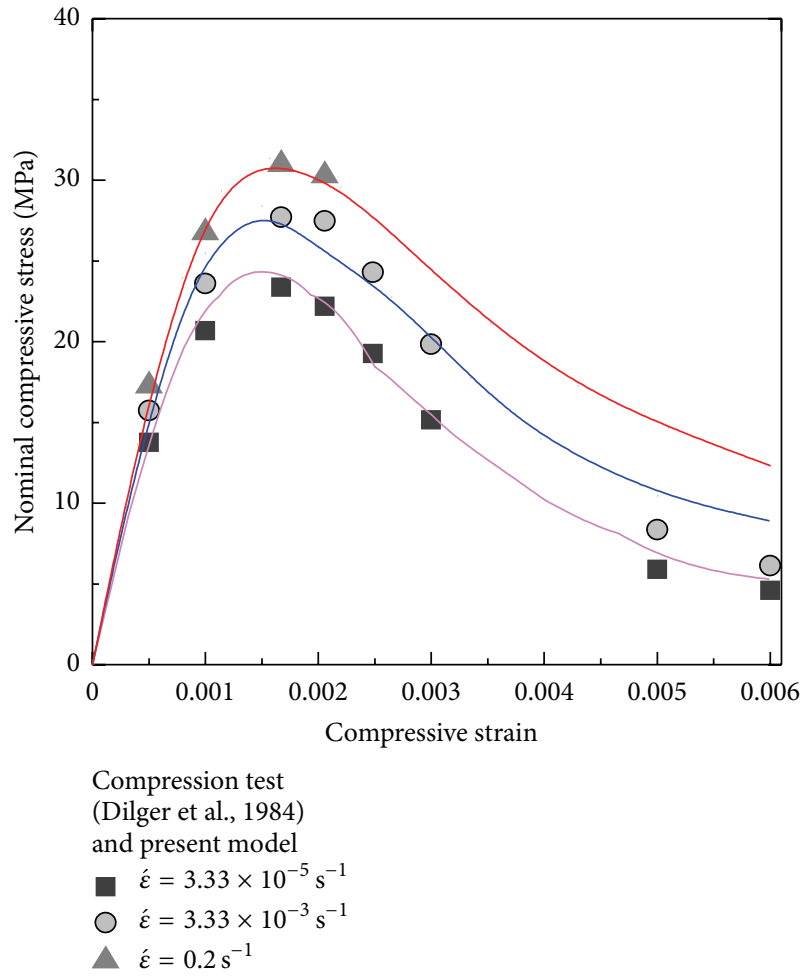

(a)

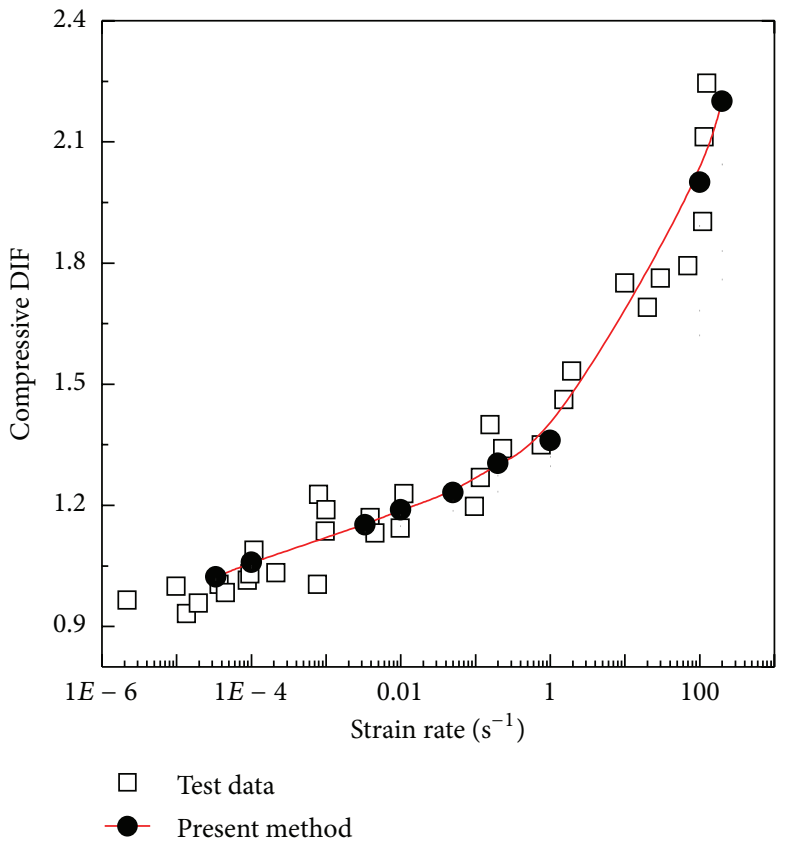

(b)

FIGURE 6: (a) Comparison of the present simulation results and Dilger et al.s [14] test data and (b) compressive DIFs for the simulation results and some available test data. 


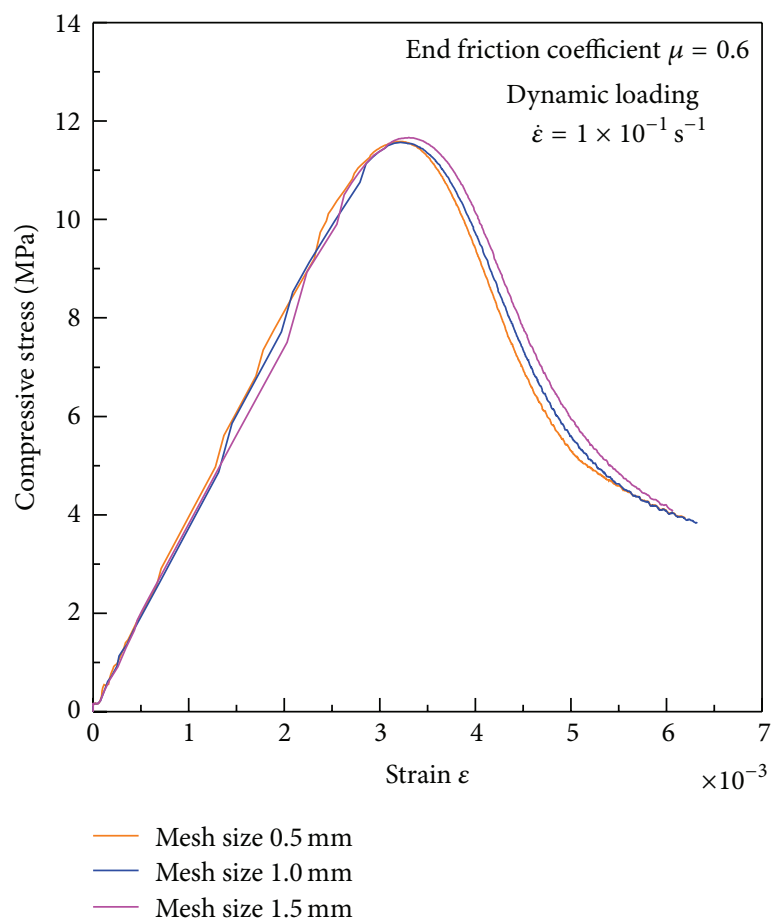

(a)

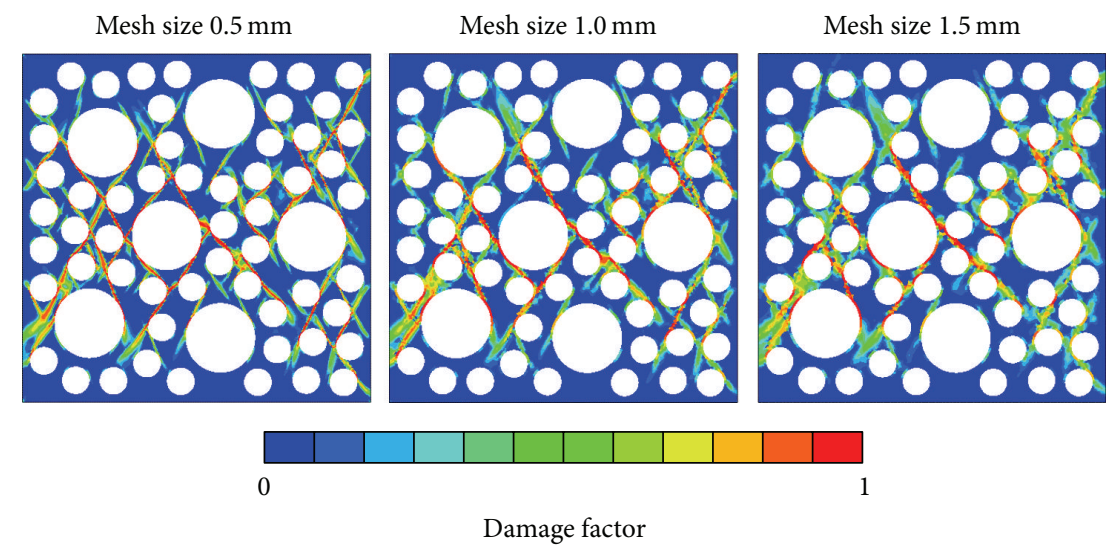

(b)

FIGURE 7: (a) The simulation results of three concrete specimens with the same distribution of aggregates and different mesh sizes and (b) damage distribution of the concrete specimens with different mesh sizes under the same strain rate and friction coefficients, at their peak stresses.

$v=1.5 \times 10^{-6} \mathrm{~m} / \mathrm{s}, 1.5 \times 10^{-4} \mathrm{~m} / \mathrm{s}, 1.5 \times 10^{-3} \mathrm{~m} / \mathrm{s}, 1.5 \times$ $10^{-2.5} \mathrm{~m} / \mathrm{s}, 1.5 \times 10^{-2} \mathrm{~m} / \mathrm{s}, 1.5 \times 10^{-1.5} \mathrm{~m} / \mathrm{s}$, and $1.5 \times 10^{-1} \mathrm{~m} / \mathrm{s}$ in an ascending sequence, and in every loading cycle the constant velocity was utilized. The corresponding macroscopic nominal strain rates are $\dot{\varepsilon}=1 \times 10^{-5} \mathrm{~s}^{-1}$ (quasistatic), $1 \times$ $10^{-3} \mathrm{~s}^{-1}, 1 \times 10^{-2} \mathrm{~s}^{-1}, 1 \times 10^{-1.5} \mathrm{~s}^{-1}, 1 \times 10^{-1} \mathrm{~s}^{-1}, 1 \times 10^{-0.5} \mathrm{~s}^{-1}$, and $1 \times 10^{0} \mathrm{~s}^{-1}$, respectively. Moreover, as mentioned previously, the seven end friction coefficients $\mu$ considered in the following simulations are 0.0 (completely smooth), $0.1,0.2$, $0.3,0.6,0.8$, and $\infty$ (i.e., the top and bottom surfaces of the specimen were fixed in the horizontal direction), respectively.

To check the mesh sensitivity in the simulations, three different mesh sizes were utilized, that is, $0.5 \mathrm{~mm}, 1.0 \mathrm{~mm}$, and
$1.5 \mathrm{~mm}$. Figure 7(a) presents the nominal compressive stressstrain curves of the concrete specimen subjected to a nominal strain rate of $0.1 \mathrm{~s}^{-1}$, with the same end friction coefficient and the three different mesh sizes. It can be found clearly from Figure 7(a) that the mesh sizes (i.e., $0.5 \mathrm{~mm}, 1.0 \mathrm{~mm}$, and $1.5 \mathrm{~mm}$ ) have less influence on the stress-strain curves. The three curves are also overlapped. Figure 7(b) shows the damage distribution of the three concrete specimens mentioned above with the same distribution of aggregates. One can notice that the failure patterns of the three specimens with different mesh sizes are closely similar to each other. Consequently, the mesh size of $1.5 \mathrm{~mm}$ was used in the following simulations. 


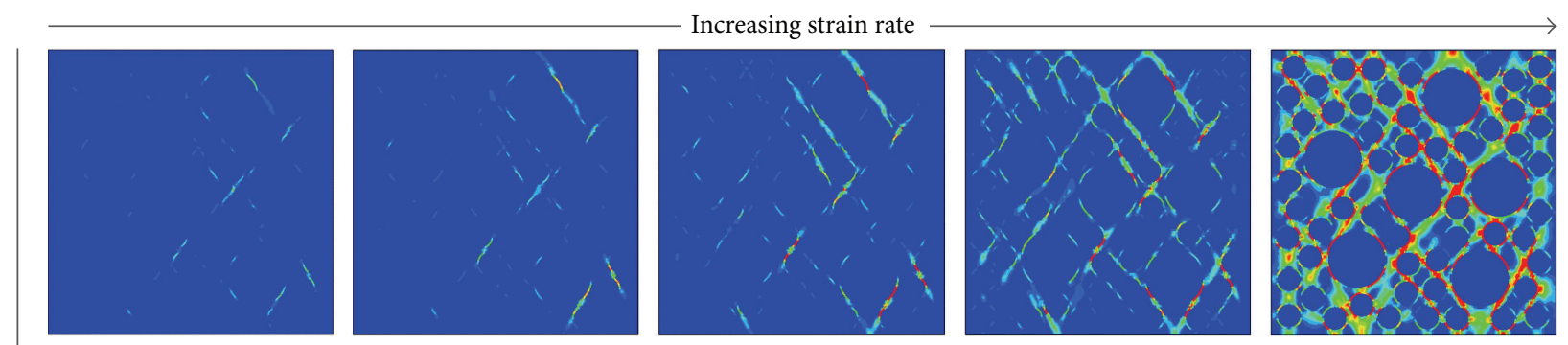

(a) $\mu=0$
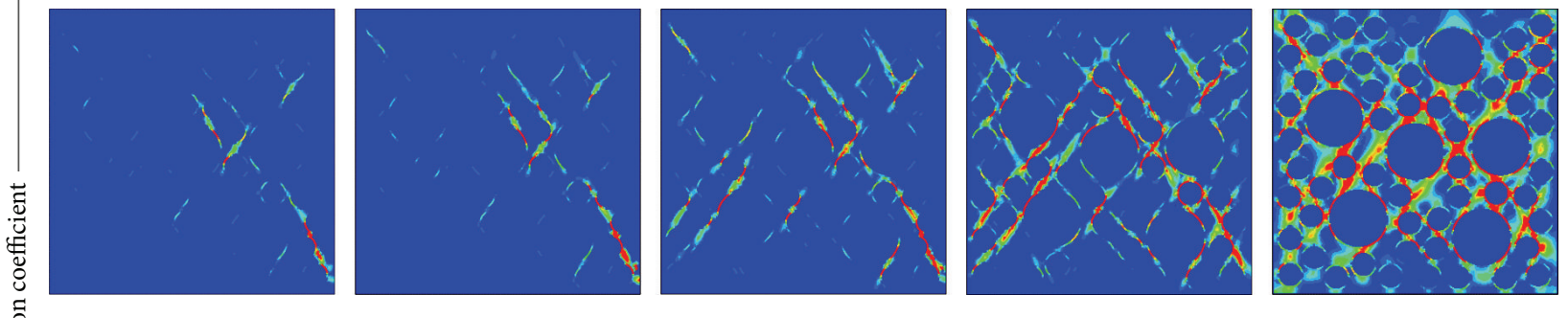

(b) $\mu=0.3$
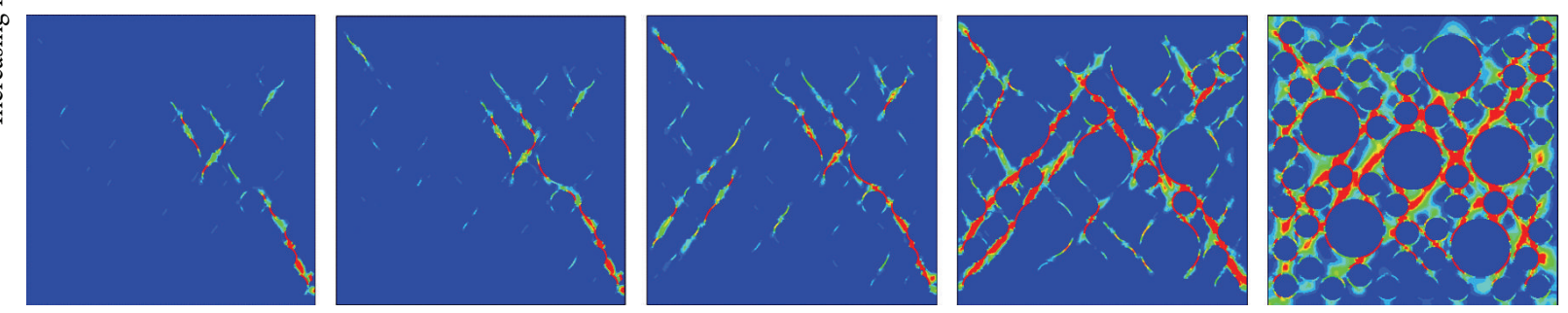

(c) $\mu=0.8$
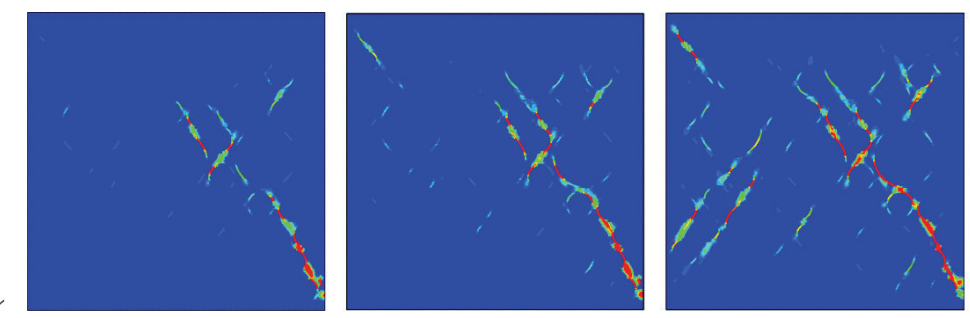

$$
\dot{\varepsilon}=1 \times 10^{-5} \mathrm{~s}^{-1}
$$

$$
\dot{\varepsilon}=1 \times 10^{-3} \mathrm{~s}^{-1}
$$$$
\dot{\varepsilon}=1 \times 10^{-2} \mathrm{~s}^{-1}
$$

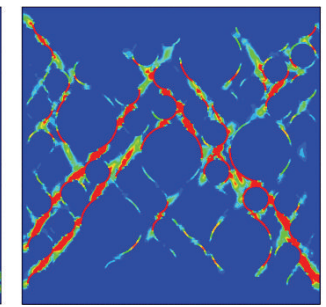

$\dot{\varepsilon}=1 \times 10^{-1} \mathrm{~s}^{-1}$

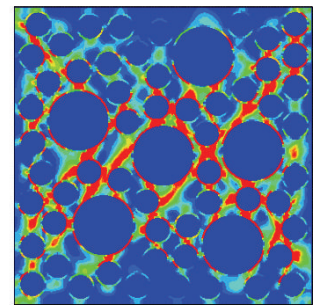

$\dot{\varepsilon}=1 \times 10^{0} \mathrm{~s}^{-1}$

0.0

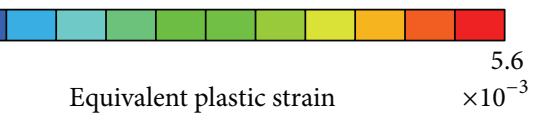

(d) $\mu=\infty$

FIGURE 8: Equivalent plastic strain distribution within the concrete specimen under different strain rates and end friction coefficients, at their peak compressive stresses.

4.1. Effects of Strain Rate and End Friction Confinement on Failure Patterns of Concrete. Figure 8 presents the equivalent plastic strain distribution within the concrete specimens under five groups of different strain rates $\left(\dot{\varepsilon}=1 \times 10^{-5} \mathrm{~s}^{-1}\right.$, $1 \times 10^{-3} \mathrm{~s}^{-1}, 1 \times 10^{-2} \mathrm{~s}^{-1}, 1 \times 10^{-1} \mathrm{~s}^{-1}$, and $\left.1 \times 10^{0} \mathrm{~s}^{-1}\right)$ and four groups of different friction coefficients $(\mu=0,0.3,0.8$, and $\infty$ ), at their peak compressive stresses (i.e., the compressive strength). One can note from Figures $8(a)-8(d)$ that, for the specimen with the same friction coefficient, with the increase of nominal strain rate, the equivalent plastic strain within concrete increases obviously. In addition, as the friction coefficient $\mu$ increases from 0 to $\infty$, the equivalent plastic strain within concrete at reaching the peak stress varies.

For the specimen with the friction coefficient of 0 and 0.3 , the obtained plastic strain distributions within the concrete specimens are obviously different. For the one with the friction coefficient of 0.3 and 0.8 , the obtained plastic strain distributions are slightly different. And, for that with the friction 


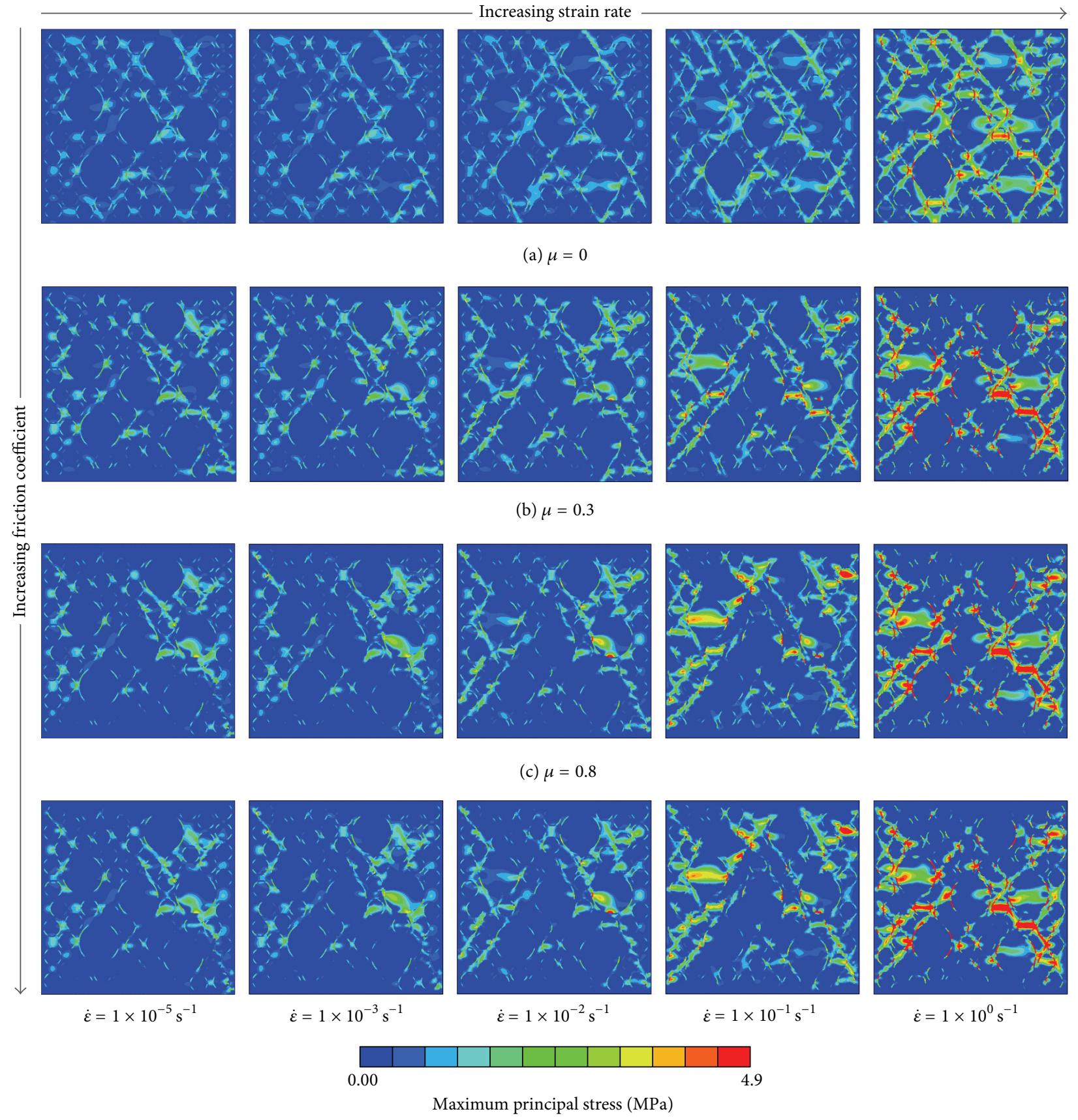

(d) $\mu=\infty$

FIGURE 9: Maximum principal stress distribution within the concrete specimen under different strain rates and end friction coefficients, at their peak compressive stresses.

coefficient of 0.8 and $\infty$, the obtained plastic strain distributions are almost totally consistent. That is to say, when the friction coefficient becomes larger than 0.8 , the effect of end friction confinement on the failure patterns of concrete can be ignored.

The maximum principal stress distributions within the concrete specimens under the five groups of different strain rates and the four different friction coefficients, at the peak compressive stresses, are presented in Figure 9. It can be noted intuitively from Figure 9 that the changes of nominal strain rate and friction coefficient have a significant influence on the maximum principal stress distribution of the specimen. With the increase of end friction coefficient, the value of maximum principal stress gets large and the distribution area draws close to central section from the top and bottom of the concrete specimen, respectively, at same loading velocity.

The damage (i.e., the cracks) distributions within the concrete specimens corresponding to Figures 8 and 9 are 

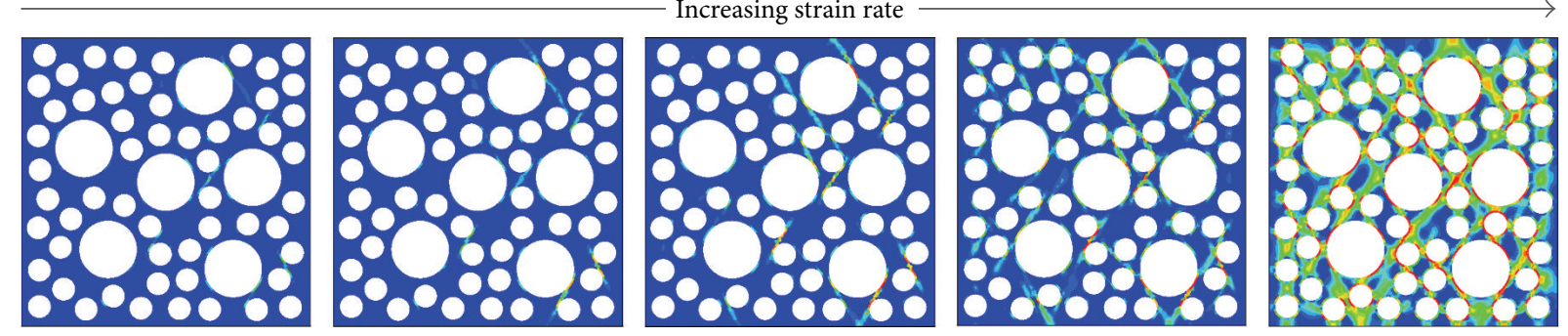

(a) $\mu=0$
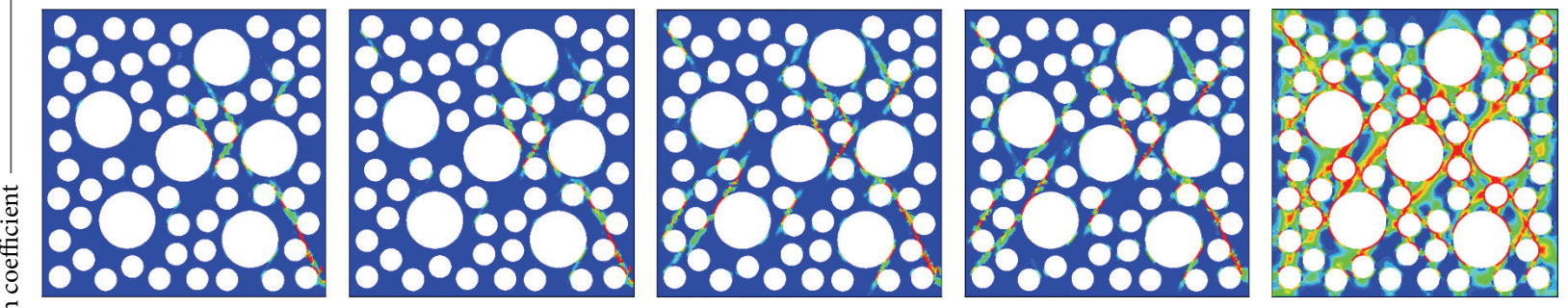

(b) $\mu=0.3$
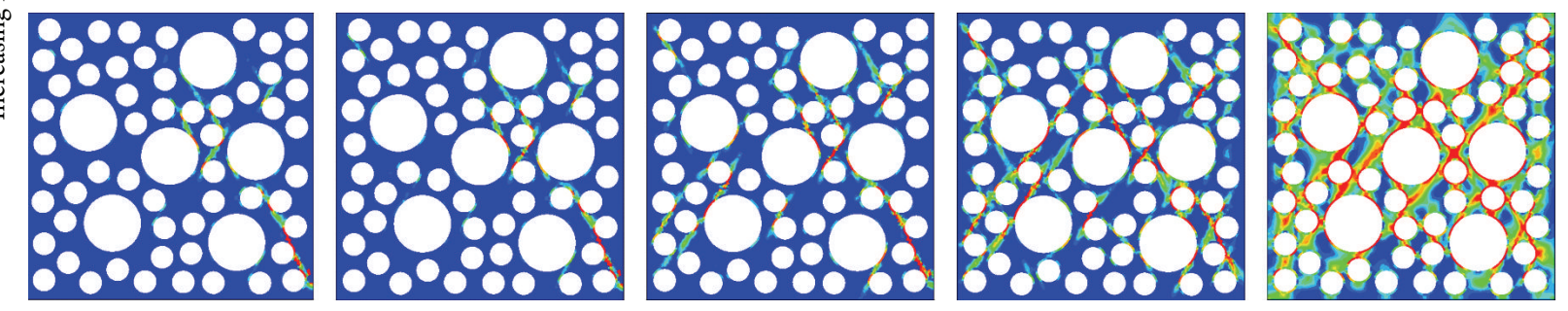

(c) $\mu=0.8$

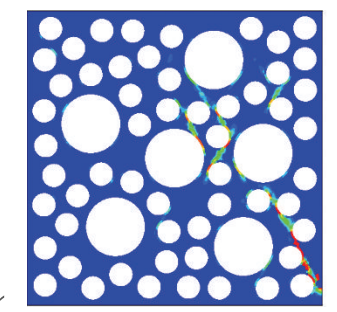

$\dot{\varepsilon}=1 \times 10^{-5} \mathrm{~s}^{-1}$

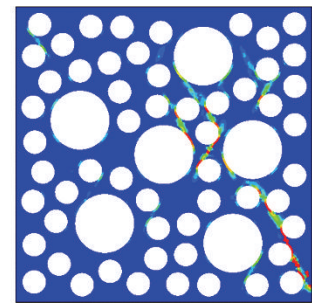

$\dot{\varepsilon}=1 \times 10^{-3} \mathrm{~s}^{-1}$

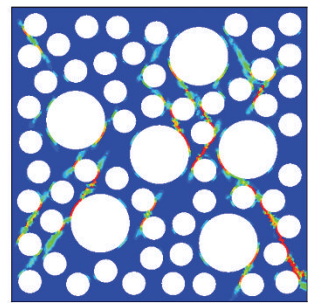

$\dot{\varepsilon}=1 \times 10^{-2} \mathrm{~s}^{-1}$

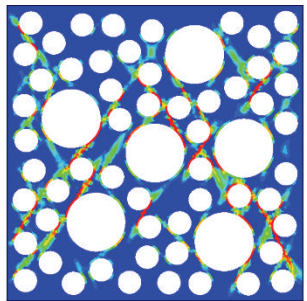

$\dot{\varepsilon}=1 \times 10^{-1} \mathrm{~s}^{-1}$

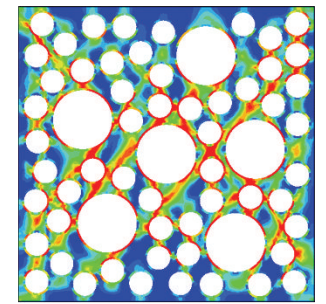

$\dot{\varepsilon}=1 \times 10^{0} \mathrm{~s}^{-1}$

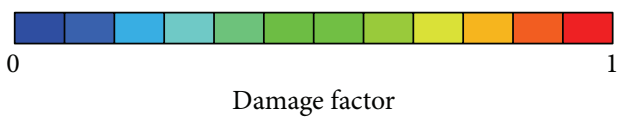

(d) $\mu=\infty$

FIGURE 10: Damage distribution within the concrete specimens under different strain rates and friction coefficients, at their peak stresses.

presented in Figure 10, for different loading velocities and different end friction coefficients. In Figure 10, different colors represent different damage indexes. The red color denotes the most serious damage, while the blue color means that no damage generates. The white rounded areas, which are regarded as aggregates in these models, have no damage.

It can be noticed clearly from Figure 10 that the damaged areas increase with the growth of nominal strain rate. When the nominal strain rate is relatively low(er) (e.g., $1 \times 10^{-5} \mathrm{~s}^{-1}$ ), the damaged area of the specimens is small when reaching their peak stress. Accordingly, the damage regions (i.e., cracks) are narrow and short, and the number of cracks is limited. As the nominal strain rate rises from $1 \times 10^{-5} \mathrm{~s}^{-1}$ to $1 \times$ $10^{0} \mathrm{~s}^{-1}$, the damaged area and the number of cracks increase gradually. When the nominal strain rate is relatively high(er) (e.g., $1 \times 10^{0} \mathrm{~s}^{-1}$ ), a lot of areas are damaged in different levels. The cracks widen and link together, forming a crack network simultaneously.

Therefore, it can be concluded that, with the increase of loading velocity, the nominal strain rate increases gradually, 
TABLE 2: Dynamic compressive strength of concrete under different end friction coefficients.

\begin{tabular}{|c|c|c|c|c|c|c|c|}
\hline \multirow[b]{2}{*}{$\mu$} & \multicolumn{7}{|c|}{ Dynamic compressive strength [MPa] } \\
\hline & $1 \times 10^{-5} \mathrm{~s}^{-1}$ & $1 \times 10^{-3} \mathrm{~s}^{-1}$ & $1 \times 10^{-2} \mathrm{~s}^{-1}$ & $1 \times 10^{-1.5} \mathrm{~s}^{-1}$ & $1 \times 10^{-1} \mathrm{~s}^{-1}$ & $1 \times 10^{-0.5} \mathrm{~s}^{-1}$ & $1 \times 10^{0} \mathrm{~s}^{-1}$ \\
\hline 0 & 7.93 & 8.06 & 8.58 & 9.19 & 10.42 & 11.97 & 15.39 \\
\hline 0.1 & 8.29 & 8.38 & 9.06 & 9.77 & 11.13 & 12.97 & 16.12 \\
\hline 0.2 & 8.46 & 8.57 & 9.26 & 10.07 & 11.37 & 13.49 & 16.82 \\
\hline 0.3 & 8.49 & 8.64 & 9.39 & 10.16 & 11.67 & 13.55 & 16.96 \\
\hline 0.6 & 8.57 & 8.77 & 9.55 & 10.36 & 11.75 & 13.82 & 17.36 \\
\hline 0.8 & 8.60 & 8.81 & 9.62 & 10.42 & 11.85 & 13.91 & 17.45 \\
\hline$\infty$ & 8.75 & 8.92 & 9.80 & 10.67 & 12.12 & 14.06 & 17.49 \\
\hline
\end{tabular}

and the number of cracks or damaged zones increases when the apparent compressive strength of concrete is reached. The energy consumption of the loading process increases correspondingly. This should be the major reason for the improvement of dynamic compressive strength of concrete materials.

It can be found obviously from the comparison among Figures 10(a)-10(d) that the damage states of concrete specimens are diverse at their peak stresses, for different end friction conditions.

Compared with the concrete specimen without end friction confinement (i.e., $\mu=0$ ), the deformation at the end of the specimen considering the effect of end friction (i.e., $\mu>0$ ) was obviously hindered. In addition, with the increase of end friction coefficient, the obstruction degree of deformation raises. For the specimen with the two ends completely fixed (i.e., $\mu=\infty$ ), there is no horizontal deformation at the ends, making the confinement effect very strong.

From Figure 10 one can note that, a larger end friction coefficient leads to a lower damage level in the top and bottom of the specimen with a constant nominal strain rate. For larger end friction coefficient, the damaged areas draw close to the central section from the top and bottom of the concrete specimen. These phenomena should generate due to the effect of the end friction confinement which changes the uniaxial loading state in the local regions of concrete. These can also be illustrated from the simulation results in Figures 8 and 9 .

\subsection{Effect of End Friction Confinement on Dynamic Compres-} sive Strength. The macroscopic nominal compressive stressstrain curves of the concrete specimen under different strain rates and end friction coefficients are plotted and compared in Figure 11. Moreover, the corresponding peak stresses, that is, the nominal dynamic compressive strengths of concrete, are reported in Table 2. Obviously, both the stress-strain curves of concrete in Figure 11 and the axial dynamic compressive strengths in Table 2 demonstrate the effects of strain rates and end friction coefficients.

It can be noted obviously from Figure 11 and Table 2 that the dynamic uniaxial compressive strength of concrete is improved due to the end friction confinement effect. Furthermore, under the same strain rates, the ascending curves of the concrete sample with various friction coefficients are almost overlapped, indicating that the elastic modulus of concrete is almost not influenced by the end friction confinement effect. The obtained compressive strengths and the softening curves are distinct significantly, because the stresses and strains status as well as the fracture paths within the concrete specimen for different friction coefficients vary.

For various strain rates, the quantitative relationships between the obtained dynamic compressive strengths of the concrete specimen and end friction coefficient are presented in Figures 12(a) and 12(b). It can be seen clearly from Figure 12 that the concrete dynamic compressive strength increases with the increase of end friction coefficient. When the end friction coefficient $\mu$ grows from 0 to 0.3 , the uniaxial compressive strength increases rapidly, while the uniaxial dynamic compressive strength increases slightly and then gets to be stable when the end friction coefficient exceeds 0.3 . This is to say, when the end friction coefficient exceeds 0.3 , the end confinement effect on the dynamic compressive mechanical behavior of concrete can be ignored. This can also be concluded from the analysis results mentioned previously, for example, the damage factor distribution of concrete presented in Figure 10.

Furthermore, the results about the effect of end friction confinement on concrete dynamic compressive strength at medium and low strain rates are similar to those at high strain rate. For more information about the end confinement effect for high strain rate refer to Li et al. [40].

Figure 13 presents the damage distribution within the concrete specimens at their corresponding peak stresses, under different end friction confinement conditions at the same loading velocities. Figure 13(a) shows the results for the loading condition of a relative lower strain rate; that is, $\dot{\varepsilon}=1 \times$ $10^{-2} \mathrm{~s}^{-1}$. One can note that, for "pure" uniaxial compressive behavior of concrete (i.e., the end friction $\mu$ between the concrete specimen and the rigid block is zero, $\mu=0$ ), the number of cracks generated or the damage degree within the specimen is very limited when reaching the compressive strength of the concrete sample. With the increase of end friction coefficient, for example, $\mu=0.1$ and 0.2 , the total number of cracks ascends gradually, but the number of cracks near the top and bottom regions of the specimen descends. When the end friction coefficient becomes larger than 0.3 (e.g., $\mu=0.3,0.6,0.8$, and $\infty$ ), the number of cracks turns to be stable, but the width of the cracks broadens, as can be seen in Figure 13(a).

Figure 13(b) shows the simulation results for the loading condition of a relative higher strain rate; that is, $\dot{\varepsilon}=1 \times$ $10^{-0.5} \mathrm{~s}^{-1}$. For "pure" uniaxial compression (i.e., the end 

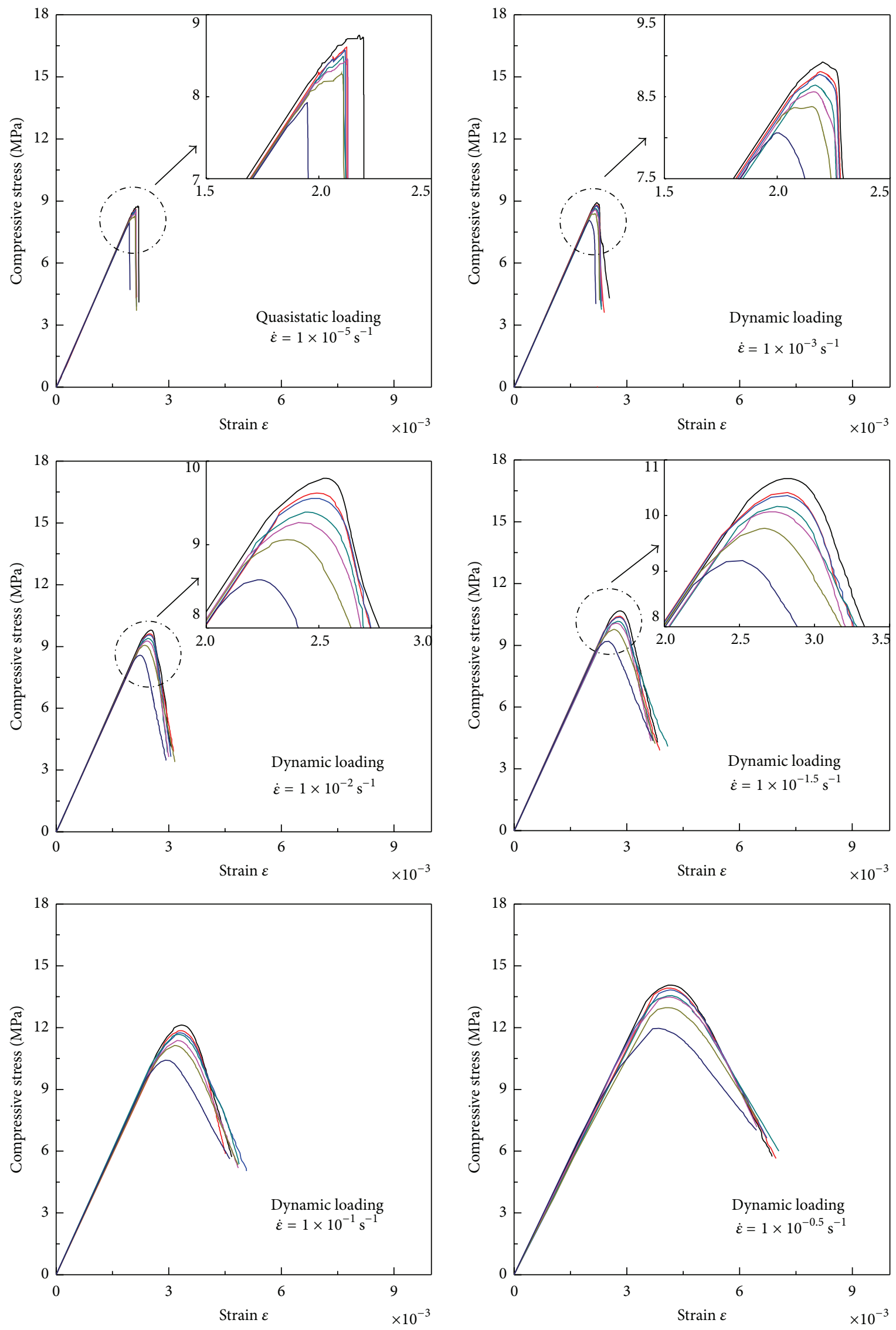

FIgURE 11: Continued. 


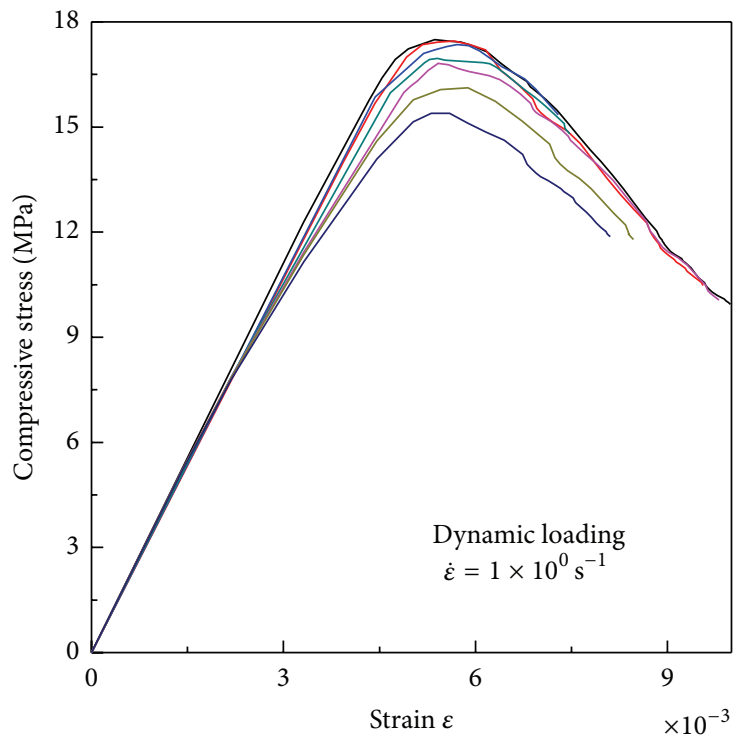

Friction coefficient

$\begin{aligned}-\mu & =\infty & -\mu & =0.2 \\ -\mu & =0.8 & -\mu & =0.1 \\ \mu & =0.6 & -\mu & =0 \\ -\mu & =0.3 & & \end{aligned}$

FIGURE 11: Dynamic compressive stress-strain curves of concrete under different strain rates and end friction coefficients.

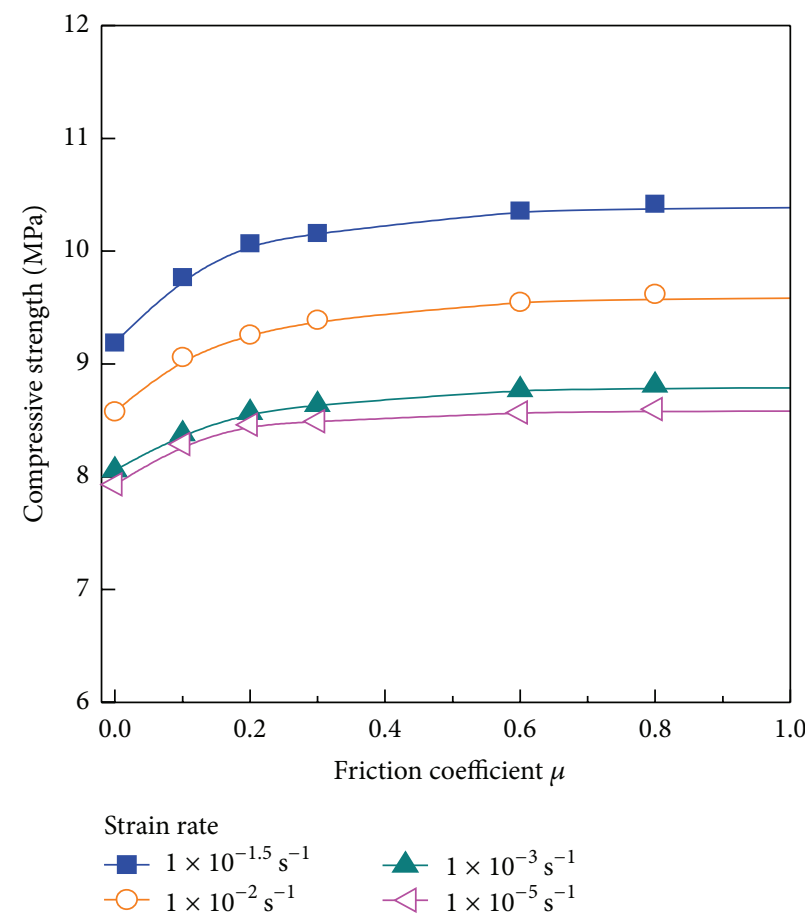

(a)

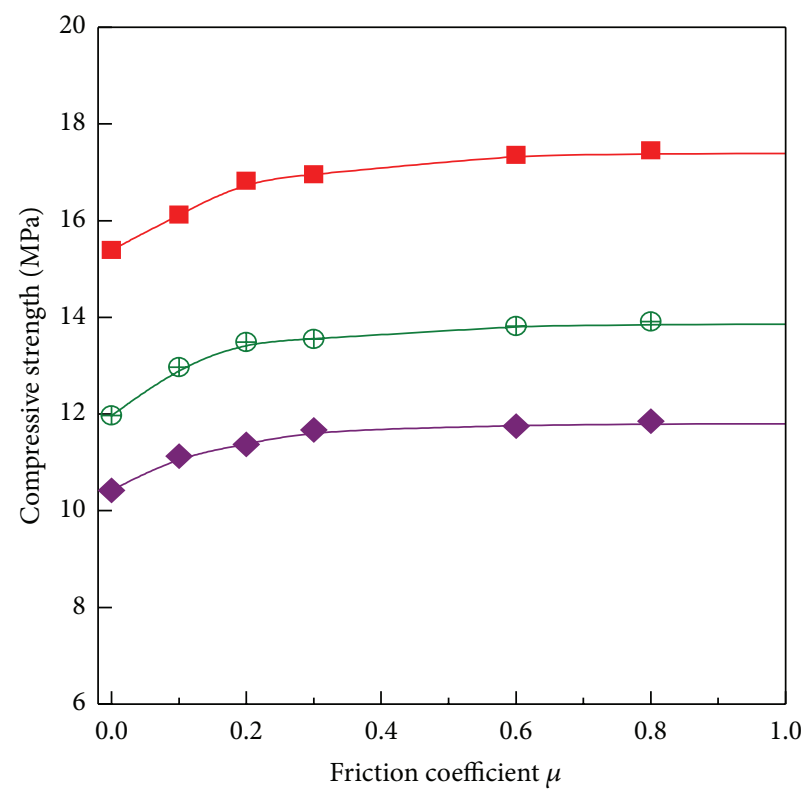

Strain rate

$-1 \times 10^{0} \mathrm{~s}^{-1}$
$-1 \times 10^{-0.5} \mathrm{~s}^{-1}$ $1 \times 10^{-1} \mathrm{~s}^{-1}$

(b)

FIGURE 12: The relationship between the obtained dynamic compressive strengths of concrete and end friction coefficient.

friction coefficient $\mu=0$ ), the cracks generated within the specimen exhibit a network distribution. With the increase of the end friction coefficient (e.g., $\mu=0.1$ and 0.2), the damage distribution concentrates on the central section from the top and bottom of the specimen. When the end friction coefficient becomes larger than 0.3 (e.g., $\mu=0.3,0.6,0.8$, and $\infty$ ), the damage distributions within the specimen become stable. For the five end friction coefficients, that is, $\mu=0.3$, 


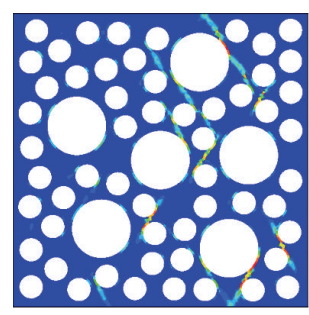

$\mu=0.0$

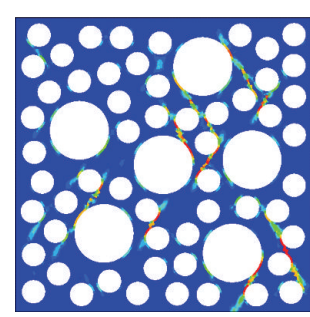

$\mu=0.1$

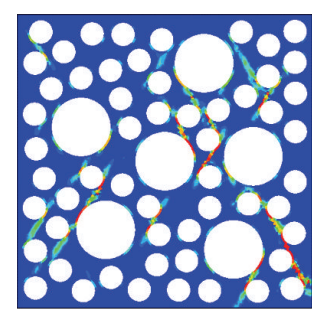

$\mu=0.2$

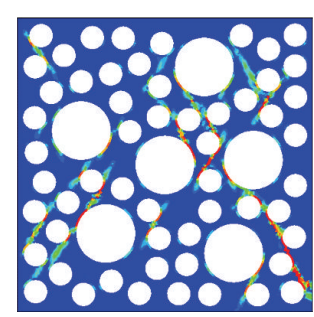

$\mu=0.3$

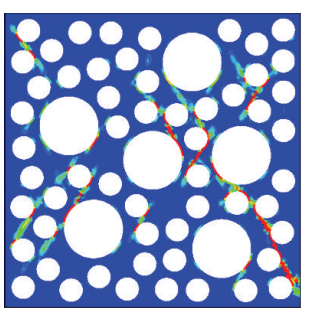

$\mu=0.6$

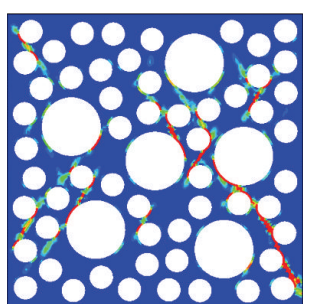

$\mu=0.8$

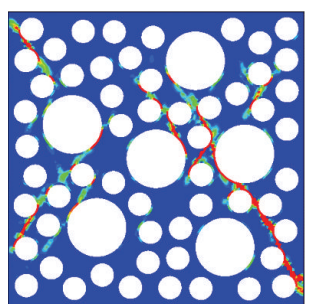

$\mu=\infty$

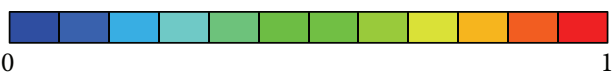

Damage factor

(a)

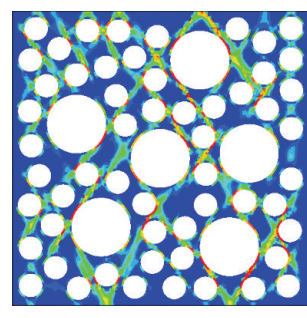

$\mu=0.0$

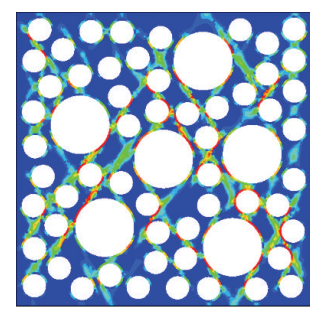

$\mu=0.1$

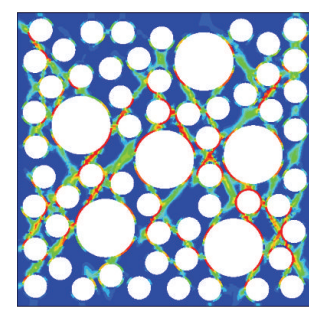

$\mu=0.2$

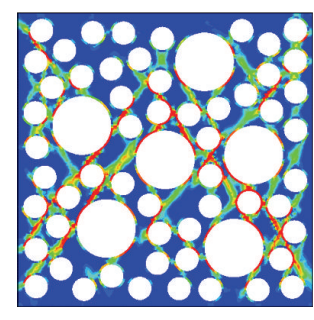

$\mu=0.3$

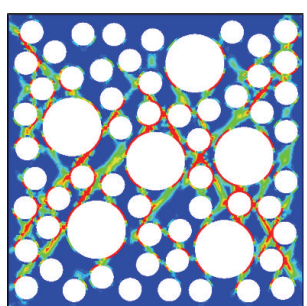

$\mu=0.6$

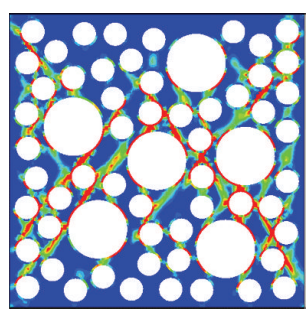

$\mu=0.8$

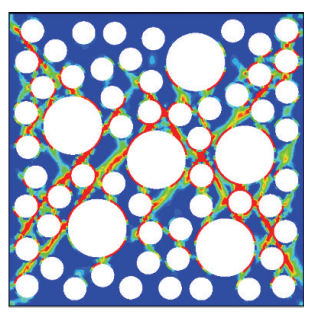

$\mu=\infty$

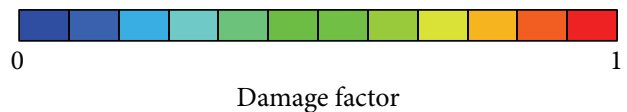

(b)

FIGURE 13: Damage distribution within concretes at their corresponding peak stresses, $(\mathrm{a})$ for $\dot{\varepsilon}=1 \times 10^{-2} \mathrm{~s}^{-1}$ and $(\mathrm{b})$ for $\dot{\varepsilon}=1 \times 10^{-0.5} \mathrm{~s}^{-1}$.

$0.6,0.8$, and $\infty$, the damage distribution and the damage degree within the specimen are almost totally the same.

According to the above analysis on the simulation results, it can be concluded that, at the medium and low strain rate levels $\left(1 \times 10^{-5} \mathrm{~s}^{-1} \sim 1 \times 10^{0} \mathrm{~s}^{-1}\right)$, the dynamic axial compressive strength of concrete increases first $(0 \leq \mu \leq 0.3)$ and then keeps stable $(\mu>0.3)$. In addition, the end friction confinement effect changes the local stress distribution with concrete and the damage pattern.
4.3. Effect of End Friction Confinement on Compressive DIFs. Li et al. [40] explored the effect of end friction confinement on the dynamic increase factor (DIF) of concrete subjected to high strain rate ranging from $27 \mathrm{~s}^{-1}$ to $390 \mathrm{~s}^{-1}$, and Hao et al. [4] explored the one ranging from $10 \mathrm{~s}^{-1}$ to $590 \mathrm{~s}^{-1}$. Herein, the present work concentrates on the one subjected to medium and low strain rate ranging from $10^{-5} \mathrm{~s}^{-1}$ to $10^{0} \mathrm{~s}^{-1}$.

Seven groups of curves which describe the relationship of compressive DIFs and nominal strain rate under different 


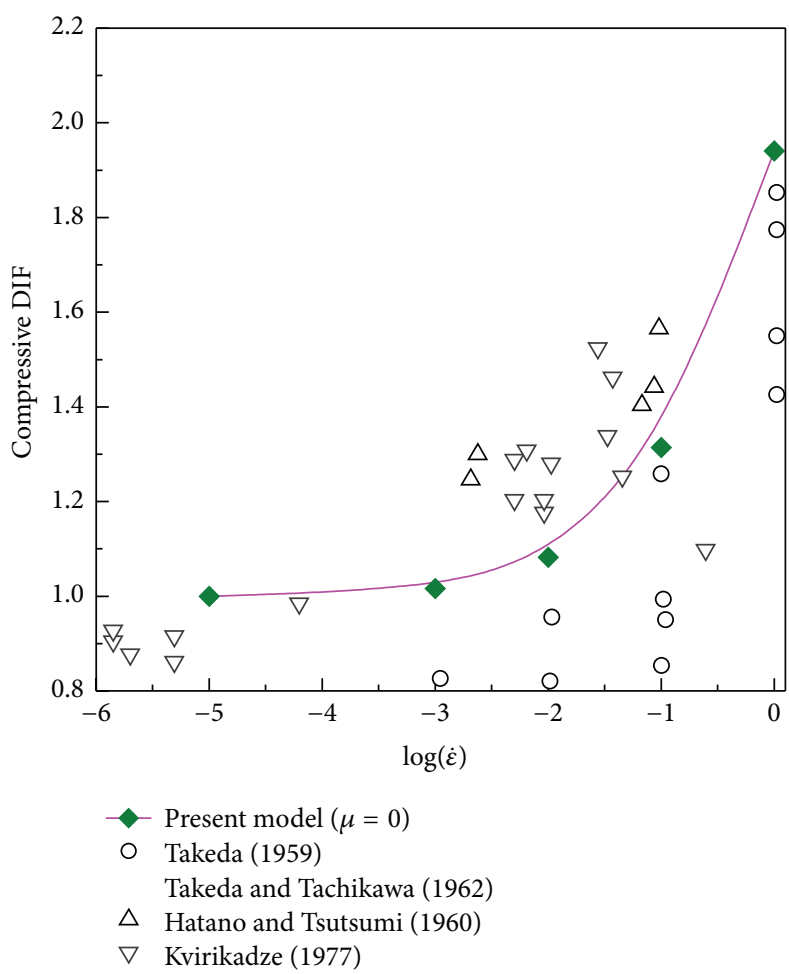

(a)

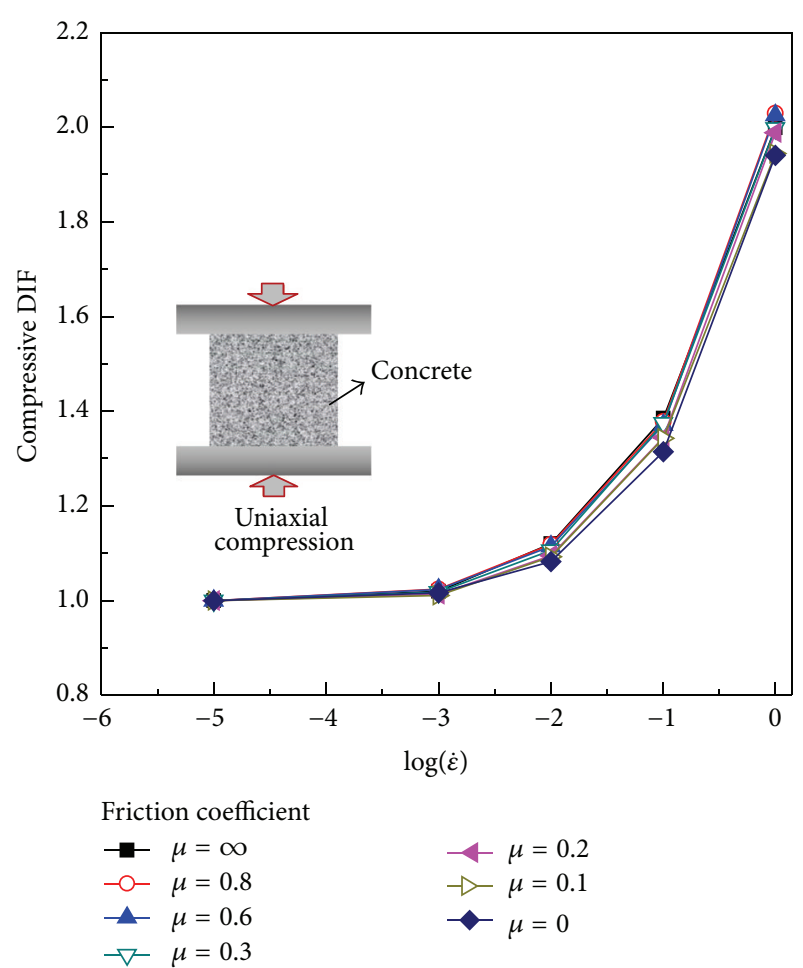

(b)

FIGURE 14: The relationship between the obtained compressive DIFs and strain rate, (a) comparison of the present simulation results and the available test data and (b) compressive DIFs of concrete under different end friction coefficients.

end friction coefficients are obtained, as shown in Figure 14. It can be found obviously from Figure 14(a) that the present simulation results for $\mu=0$ coincide well with the available experimental observations. The feasibility of the present mesomechanical method is therefore demonstrated again. Figure 14(b) plots the obtained compressive DIFs of concrete possessing various end friction coefficients. For the nominal strain rate $\dot{\varepsilon} \leq 1 \times 10^{-3} \mathrm{~s}^{-1}$, the dynamic compressive strength of concrete improves slightly. While for the nominal strain rate $\dot{\varepsilon} \geq 1 \times 10^{-2} \mathrm{~s}^{-1}$, the dynamic compressive strength increases rapidly. Exactly for this, the effect of end friction confinement on the compressive DIFs of concrete with the nominal strain rate of $\dot{\varepsilon} \geq 1 \times 10^{-2} \mathrm{~s}^{-1}$ draws much attention.

To examine the quantitative effect of end friction confinement, a new factor $\beta_{\mu \dot{\varepsilon}}$, contribution of end friction confinement in the improvement of concrete dynamic axial compressive strength, is given in the form of the following equation:

$$
\beta_{\mu \dot{\varepsilon}}=\frac{\mathrm{DIF}_{\mu \dot{\varepsilon}}-\mathrm{DIF}_{0 \dot{\varepsilon}}}{\mathrm{DIF}_{\mu \dot{\varepsilon}}-1}
$$

where the subscript $\mu$ is the end friction coefficient; $\dot{\varepsilon}$ means the nominal strain rate; $\mathrm{DIF}_{\mu \dot{\varepsilon}}$ is the dynamic increase factor for the end friction coefficient $\mu$ and the nominal strain rate $\dot{\varepsilon}$; $\mathrm{DIF}_{0 \dot{\varepsilon}}$ is the dynamic increase factor when the end friction coefficient $\mu$ is equal to zero (i.e., without considering the effect of end friction confinement); and the nominal strain rate is $\dot{\varepsilon}$.
Figure 15(a) shows the quantitative relationships between the friction contribution factor $\beta$ and end friction coefficient $\mu$ under different nominal strain rates $\dot{\varepsilon}$. It can be seen from Figure 15(a) that when the end friction coefficient $\mu$ grows from 0.1 to 0.3 , the friction contribution factor $\beta$ increases significantly. However, as the end friction coefficient grows from 0.3 to $\infty$, the friction contribution factor increases slightly and then gets to be stable. In addition, one can see from Figure 15(a) that, with the increase of strain rate, the friction contribution factor decreases especially when the friction coefficient exceeds 0.3 .

Figure 15(b) plots the relationship between friction contribution factor $\beta$ and nominal strain rate (described by $\log (\dot{\varepsilon}))$ under different end friction confinement conditions. With the increase of nominal strain rate, the friction contribution factor presents a descending trend. Moreover, with the increase of end friction coefficient, the descending trend gets to be much more obvious. When the strain rate is relatively lower (i.e., for $\dot{\varepsilon}=10^{-2} \mathrm{~s}^{-1}$ ), the larger the end friction coefficient is, the larger the friction contribution factor is.

For further research, considering the extreme situation of end confinement, that is, $\mu=\infty$, the ascending parts of the axial dynamic compressive stress-strain relationship curves of the concrete specimens are obtained, as shown in Figure 16, for the relatively low strain rate $\dot{\varepsilon}=1 \times 10^{-2} \mathrm{~s}^{-1}$ and the relatively high strain rate $\dot{\varepsilon}=1 \times 10^{0} \mathrm{~s}^{-1}$. In Figure 16, $E_{1}$ denotes the energy consumption when the concrete specimen without considering the effect of end friction confinement (i.e., $\mu=0$ ) 


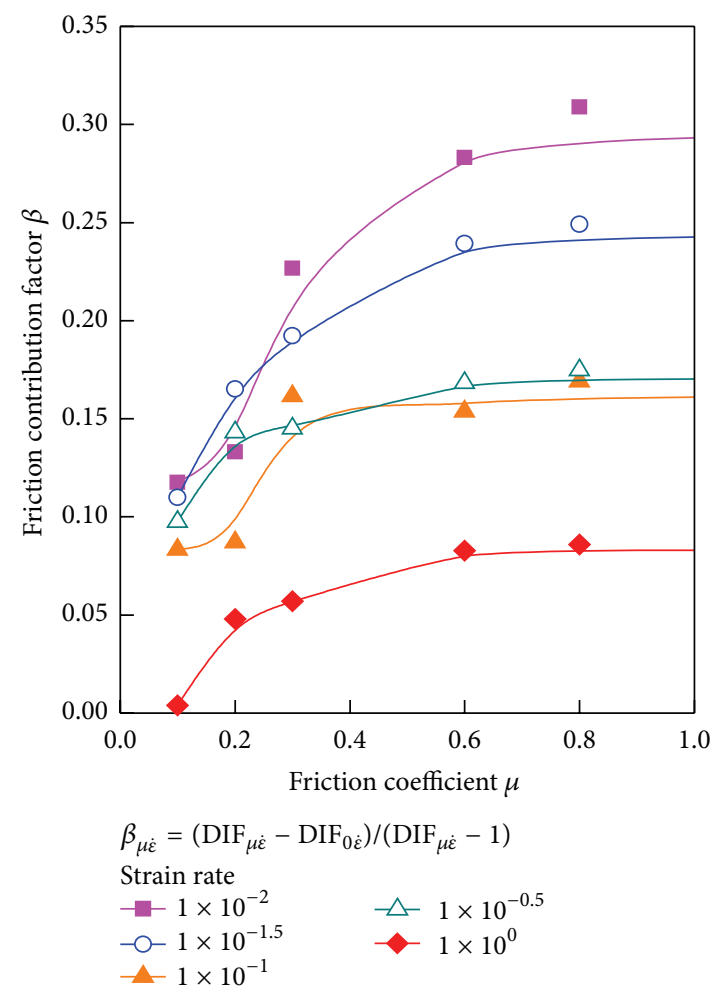

(a)

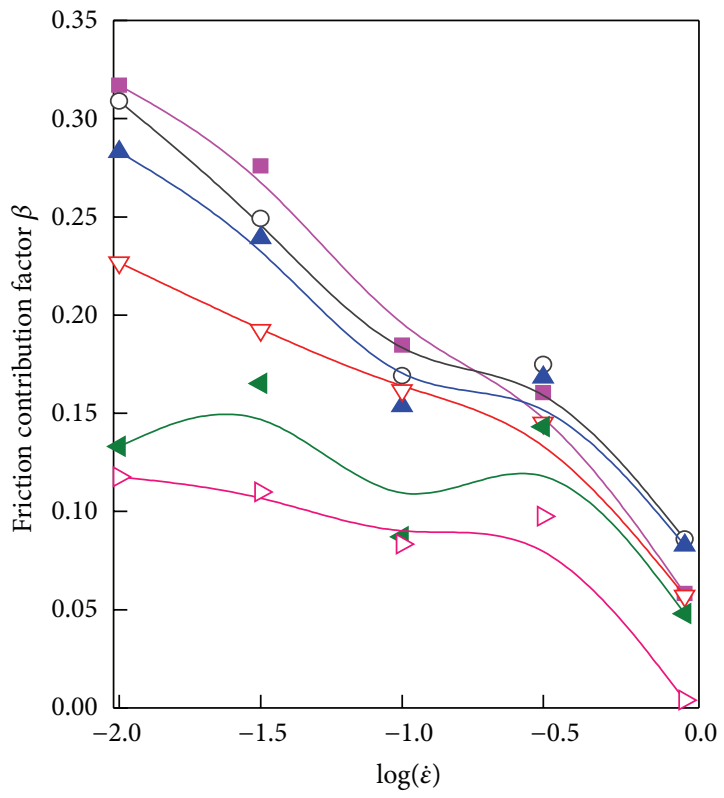

$\beta_{\mu \dot{\varepsilon}}=\left(\mathrm{DIF}_{\mu \dot{\varepsilon}}-\mathrm{DIF}_{0 \dot{\varepsilon}}\right) /\left(\mathrm{DIF}_{\mu \dot{\varepsilon}}-1\right)$ Friction coefficient

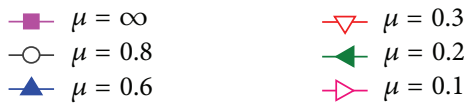

(b)

FIGURE 15: Effects of friction coefficient $\mu$ and strain rate $\dot{\varepsilon}$ on the friction contribution factor $\beta$, (a) $\beta$ and $\mu$ and (b) $\beta$ and $\dot{\varepsilon}$.

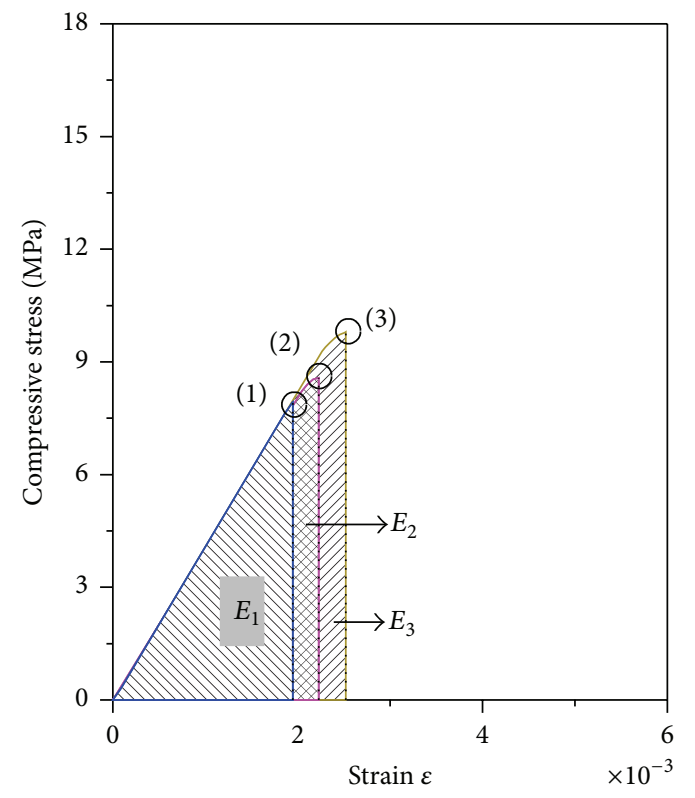
$\begin{array}{cc}\text { Friction coefficient } & \text { (1) Quasistatic loading } \\ -(3) \mu=\infty & \dot{\varepsilon}=1 \times 10^{-5} \mathrm{~s}^{-1}\end{array}$

- (2) $\mu=0$ - (1) $\mu=0$

(2), (3) Dynamic loading $\dot{\varepsilon}=1 \times 10^{-2} \mathrm{~s}^{-1}$

(a)

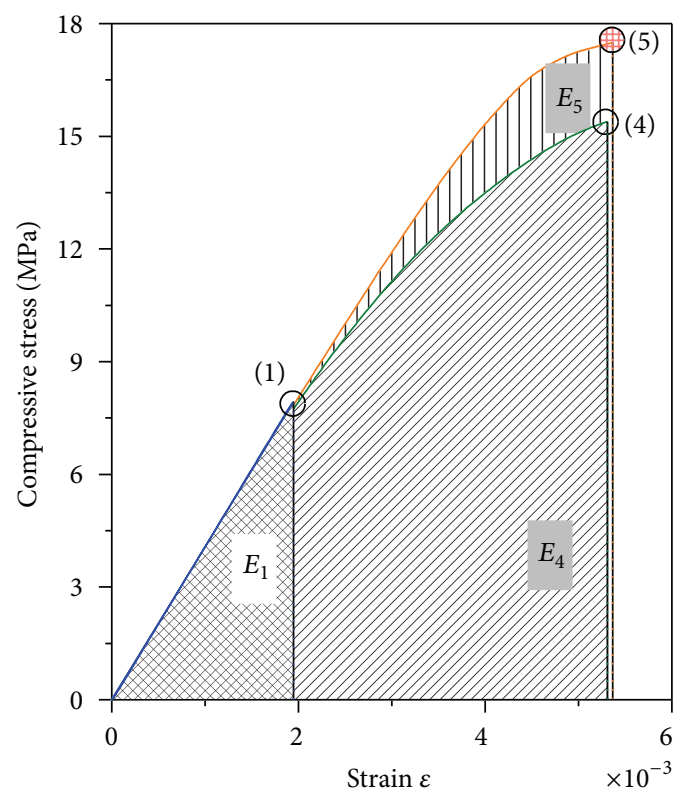

$\begin{array}{ll}\text { Friction coefficient } & \text { (1) Quasistatic loading } \\ \dot{\varepsilon}=1 \times 10^{-5} \mathrm{~s}^{-1}\end{array}$

- (4) $\mu=0$

- (1) $\mu=0$

(4), (5) Dynamic loading $\dot{\varepsilon}=1 \times 10^{0} \mathrm{~s}^{-1}$

(b)

FIGURE 16: The ascending part of concrete dynamic uniaxial compressive stress-strain curves, (a) for a lower strain rate of $1 \times 10^{-2} \mathrm{~s}^{-1}$ and (b) for a higher strain rate of $1 \times 10^{-0} \mathrm{~s}^{-1}$. 
reaches its quasistatic nominal compressive strength (the strain rate $\dot{\varepsilon}=1 \times 10^{-5} \mathrm{~s}^{-1}$ ), and its magnitude is equal to the area occupied by the ascending curve and the horizontal axial; both $E_{2}$ and $E_{4}$ denote the energy consumption for the strength improvement due to material strain rate effect; $E_{3}$ and $E_{5}$ denote the energy consumption for the strength improvement due to end friction confinement. It can be seen clearly from Figure 16 that

$$
\frac{E_{3}}{E_{2}+E_{3}}>\frac{E_{5}}{E_{4}+E_{5}} .
$$

That is to say, when the nominal strain rate is relatively low(er) (e.g., $\dot{\varepsilon}=1 \times 10^{-2} \mathrm{~s}^{-1}$ ), the contribution of end friction confinement effect to the enhancement of dynamic compressive strength is relatively high(er). While the nominal strain rate is relatively high(er) (e.g., $\dot{\varepsilon}=1 \times 10^{0} \mathrm{~s}^{-1}$ ), the contribution of end friction confinement effect to dynamic strength improvement is relatively low(er). The simulation results are consistent with those in the work [4].

\section{Conclusions}

Considering the heterogeneities, two-dimensional mesoscale mechanical models of concrete were established to investigate the quantitative influence of end friction confinement on the dynamic compressive behavior of concrete specimens under medium and low strain rates. The proposed mesoscale analysis method was firstly verified by comparing the present simulation results with the available test data. In the simulations, seven groups of friction coefficients, namely, 0.0, 0.1, $0.2,0.3,0.6,0.8$, and $\infty$, are taken into account. The effects of end friction confinement on the dynamic failure patterns, the nominal compressive stress-strain relations, and the compressive DIFs were explored subsequently. One can find that the friction at the interface of concrete specimen and loading measurement constrains the lateral deformation of the specimen and changes the internal stress status, and thus it has a significant influence on the tested DIF. Furthermore, the friction contribution factor was proposed and it was used to describe the quantitatively influence of end friction confinement. According to the simulations, the following conclusions can be obtained.

(1) At the medium and low strain rate level $(1 \times$ $\left.10^{-5} \mathrm{~s}^{-1} \sim 1 \times 10^{0} \mathrm{~s}^{-1}\right)$, the uniaxial dynamic compressive strength increases first $(0 \leq \mu \leq 0.3)$ and then keeps stable $(\mu>0.3)$.

(2) End friction confinement effect changes the uniaxial force state of local concrete and the failure patterns. Meanwhile, the concrete uniaxial dynamic compressive strength is improved due to the end friction confinement effect.

(3) For same end friction confinement, the friction contribution factor decreases with the increase of nominal strain rate (ranging from $1 \times 10^{-2} \mathrm{~s}^{-1}$ to $1 \times 10^{0} \mathrm{~s}^{-1}$ ). And with the increase of end friction coefficient, the descending trend gets to be obvious.
It is to be noted that further studies are still necessary for a fully understanding about the strain-rate effect on the ultimate uniaxial compressive strength of concrete and concretelike materials subjected to dynamic loadings. Both tests and simulations could offer more information to separate pseudostrain-rate effects from genuine strain-rate effects in dynamic material properties tests, which is of significant importance for material model identification.

\section{Conflict of Interests}

The authors declare that there is no conflict of interests regarding the publication of this paper.

\section{Acknowledgments}

This work was supported by the National Key Basic Research and Development Program of China (no. 2011CB013600) and the Science Fund for Creative Research Groups of the National Natural Science Foundation of China (no. 51421005). The support is gratefully acknowledged.

\section{References}

[1] H. Xu and H. M. Wen, "Semi-empirical equations for the dynamic strength enhancement of concrete-like materials," International Journal of Impact Engineering, vol. 60, pp. 76-81, 2013.

[2] Q. M. Li and H. Meng, "About the dynamic strength enhancement of concrete-like materials in a split Hopkinson pressure bar test," International Journal of Solids and Structures, vol. 40, no. 2, pp. 343-360, 2003.

[3] P. H. Bischoff and S. H. Perry, "Compressive behaviour of concrete at high strain rates," Materials and Structures, vol. 24, no. 6, pp. 425-450, 1991.

[4] Y. Hao, H. Hao, and Z. X. Li, "Influence of end friction confinement on impact tests of concrete material at high strain rate," International Journal of Impact Engineering, vol. 60, pp. 82-106, 2013.

[5] M. J. Forrestal, T. W. Wright, and W. Chen, "The effect of radial inertia on brittle samples during the split Hopkinson pressure bar test," International Journal of Impact Engineering, vol. 34, no. 3, pp. 405-411, 2007.

[6] Z. C. Mu, A. N. Dancygier, W. Zhang, and D. Z. Yankelevsky, "Revisiting the dynamic compressive behavior of concrete-like materials," International Journal of Impact Engineering, vol. 49, pp. 91-102, 2012.

[7] D. J. Kim, K. Sirijaroonchai, S. E. El-Tawil, and A. Naaman, "Numerical simulation of the split Hopkinson pressure bar test technique for concrete under compression," International Journal of Impact Engineering, vol. 37, no. 2, pp. 141-149, 2010.

[8] R. R. Pedersen, A. Simone, and L. J. Sluys, "Mesoscopic modeling and simulation of the dynamic tensile behavior of concrete," Cement and Concrete Research, vol. 50, pp. 74-87, 2013.

[9] X. Q. Zhou and H. Hao, "Mesoscale modelling of concrete tensile failure mechanism at high strain rates," Computers and Structures, vol. 86, no. 21-22, pp. 2013-2026, 2008.

[10] X. Q. Zhou and H. Hao, "Modelling of compressive behaviour of concrete-like materials at high strain rate," International Journal of Solids and Structures, vol. 45, no. 17, pp. 4648-4661, 2008. 
[11] X. L. Du, L. Jin, and G. W. Ma, "Numerical simulation of dynamic tensile-failure of concrete at meso-scale," International Journal of Impact Engineering, vol. 66, pp. 5-17, 2014.

[12] X. L. Du, L. Jin, and R. B. Zhang, "Modeling the cracking of cover concrete due to non-uniform corrosion of reinforcement," Corrosion Science, vol. 89, pp. 189-202, 2014.

[13] S.-M. Kim and R. K. Abu Al-Rub, "Meso-scale computational modeling of the plastic-damage response of cementitious composites," Cement and Concrete Research, vol. 41, no. 3, pp. 339358, 2011.

[14] W. H. Dilger, R. Koch, and R. Kowalczyk, "Ductility of plain and confined concrete under different strain rates," ACI Journal, vol. 81, no. 1, pp. 73-81, 1984.

[15] J. Lubliner, J. Oliver, S. Oller, and E. Oñate, "A plastic-damage model for concrete," International Journal of Solids and Structures, vol. 25, no. 3, pp. 299-326, 1989.

[16] J. Lee and G. L. Fenves, "Plastic-damage model for cyclic loading of concrete structures," Journal of Engineering Mechanics, vol. 124, no. 8, pp. 892-900, 1998.

[17] P. Grassl and M. Jirásek, "Damage-plastic model for concrete failure," International Journal of Solids and Structures, vol. 43, no. 22-23, pp. 7166-7196, 2006.

[18] P. Badel, V. Godard, and J.-B. Leblond, "Application of some anisotropic damage model to the prediction of the failure of some complex industrial concrete structure," International Journal of Solids and Structures, vol. 44, no. 18-19, pp. 5848-5874, 2007.

[19] Y. Huang, Z. Yang, W. Ren, G. Liu, and C. Zhang, "3D mesoscale fracture modelling and validation of concrete based on in-situ X-ray computed tomography images using damage plasticity model," International Journal of Solids and Structures, vol. 67-68, pp. 340-352, 2015.

[20] A. S. Genikomsou and M. A. Polak, "Finite element analysis of punching shear of concrete slabs using damaged plasticity model in ABAQUS," Engineering Structures, vol. 98, pp. 38-48, 2015.

[21] D. L. Grote, S. W. Park, and M. Zhou, "Dynamic behavior of concrete at high strain rates and pressures: I. Experimental characterization," International Journal of Impact Engineering, vol. 25, no. 9, pp. 869-886, 2001.

[22] S. W. Park, Q. Xia, and M. Zhou, "Dynamic behavior of concrete at high strain rates and pressures. II. Numerical simulation," International Journal of Impact Engineering, vol. 25, no. 9, pp. 887-910, 2001.

[23] S. Hentz, F. V. Donzé, and L. Daudeville, "Discrete element modelling of concrete submitted to dynamic loading at high strain rates," Computers \& Structures, vol. 82, no. 29-30, pp. 2509-2524, 2004.

[24] L. J. Malvar and C. A. Ross, "Review of strain rate effects for concrete in tension," ACI Materials Journal, vol. 95, no. 6, pp. 735-739, 1998.

[25] X. Q. Zhou, V. A. Kuznetsov, H. Hao, and J. Waschl, "Numerical prediction of concrete slab response to blast loading," International Journal of Impact Engineering, vol. 35, no. 10, pp. 11861200, 2008.

[26] G. Cusatis, "Strain-rate effects on concrete behavior," International Journal of Impact Engineering, vol. 38, no. 4, pp. 162-170, 2011.

[27] J. Ožbolt, A. Sharma, and H.-W. Reinhardt, "Dynamic fracture of concrete-compact tension specimen," International Journal of Solids and Structures, vol. 48, no. 10, pp. 1534-1543, 2011.
[28] S. Pyo and S. El-Tawil, "Crack velocity-dependent dynamic tensile behavior of concrete," International Journal of Impact Engineering, vol. 55, pp. 63-70, 2013.

[29] N. Tregger, D. Corr, L. Graham-Brady, and S. Shah, "Modeling the effect of mesoscale randomness on concrete fracture," Probabilistic Engineering Mechanics, vol. 21, no. 3, pp. 217-225, 2006.

[30] W. Riedel, M. Wicklein, and K. Thoma, "Shock properties of conventional and high strength concrete: experimental and mesomechanical analysis," International Journal of Impact Engineering, vol. 35, no. 3, pp. 155-171, 2008.

[31] F. Fakhari Tehrani, J. Absi, F. Allou, and C. Petit, "Heterogeneous numerical modeling of asphalt concrete through use of a biphasic approach: porous matrix/inclusions," Computational Materials Science, vol. 69, pp. 186-196, 2013.

[32] F. Bernard and S. Kamali-Bernard, "Numerical study of ITZ contribution on mechanical behavior and diffusivity of mortars," Computational Materials Science, vol. 102, pp. 250-257, 2015.

[33] L. Jin and X.-L. Du, "Effects of loading rate and its sudden change on concrete compressive failure," Journal of Vibration and Shock, vol. 33, no. 19, pp. 187-193, 2014.

[34] X. L. Du, L. Jin, and G. W. Ma, "A meso-scale analysis method for the simulation of nonlinear damage and failure behavior of reinforced concrete members," International Journal of Damage Mechanics, vol. 22, no. 6, pp. 878-904, 2013.

[35] L. Snozzi, A. Caballero, and J. F. Molinari, "Influence of the meso-structure in dynamic fracture simulation of concrete under tensile loading," Cement and Concrete Research, vol. 41, no. 11, pp. 1130-1142, 2011.

[36] H.-K. Man and J. G. M. van Mier, "Damage distribution and size effect in numerical concrete from lattice analyses," Cement and Concrete Composites, vol. 33, no. 9, pp. 867-880, 2011.

[37] W. Y. Ren, Z. J. Yang, R. Sharma, C. H. Zhang, and P. J. Withers, "Two-dimensional X-ray CT image based meso-scale fracture modelling of concrete," Engineering Fracture Mechanics, vol. 133, pp. 24-39, 2015.

[38] Y. Hao, H. Hao, and X. H. Zhang, "Numerical analysis of concrete material properties at high strain rate under direct tension," International Journal of Impact Engineering, vol. 39, no. 1, pp. 51-62, 2012.

[39] Y. Hao and H. Hao, "Numerical investigation of the dynamic compressive behaviour of rock materials at high strain rate," Rock Mechanics and Rock Engineering, vol. 46, no. 2, pp. 373388, 2013.

[40] Q. M. Li, Y. B. Lu, and H. Meng, "Further investigation on the dynamic compressive strength enhancement of concrete-like materials based on split Hopkinson pressure bar tests. Part II: numerical simulations," International Journal of Impact Engineering, vol. 36, no. 12, pp. 1335-1345, 2009. 


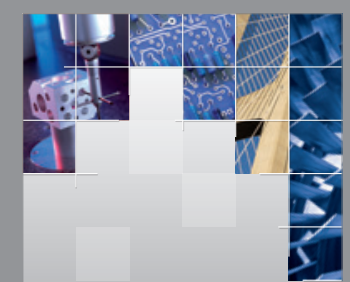

\section{Enfincering}
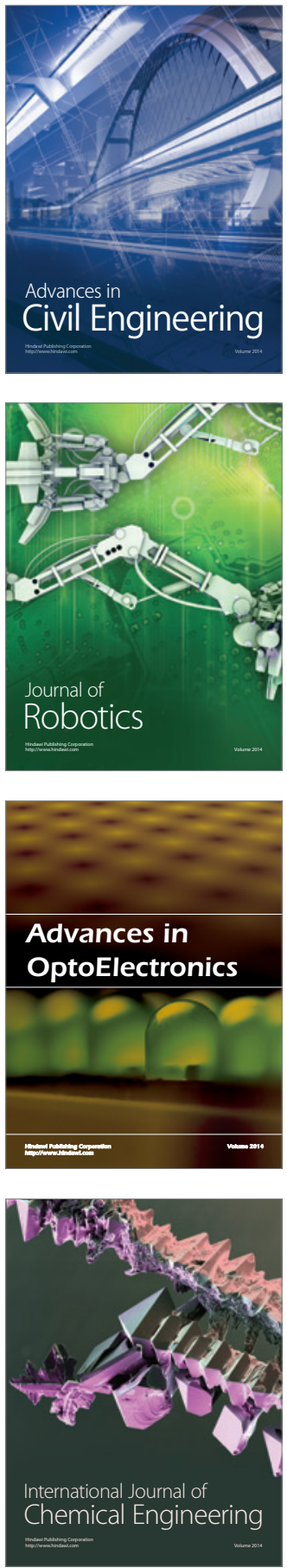

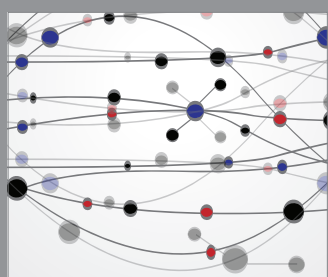

The Scientific World Journal

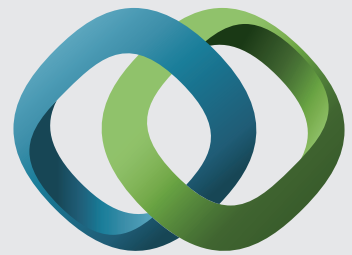

\section{Hindawi}

Submit your manuscripts at

http://www.hindawi.com
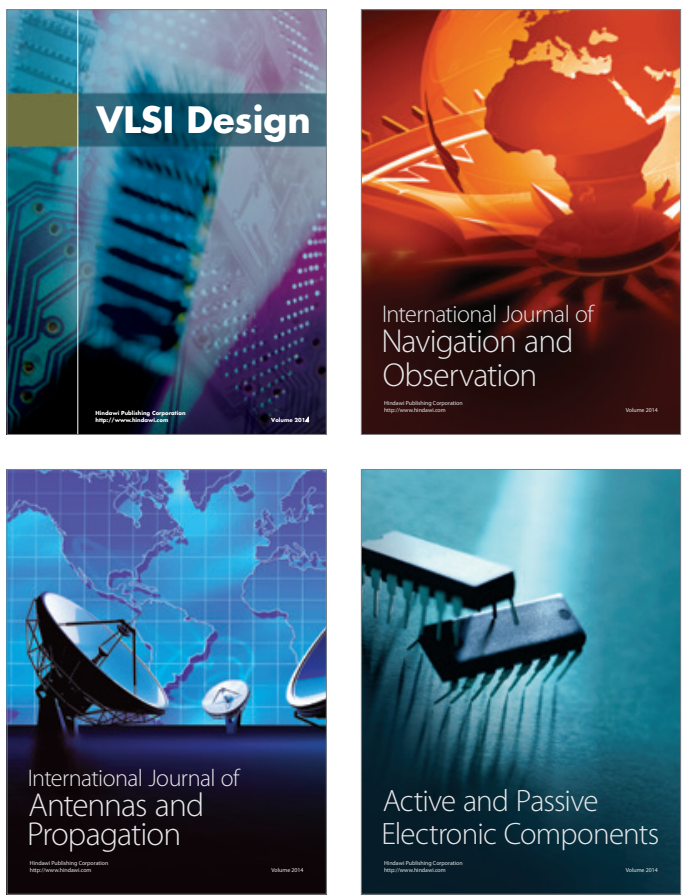
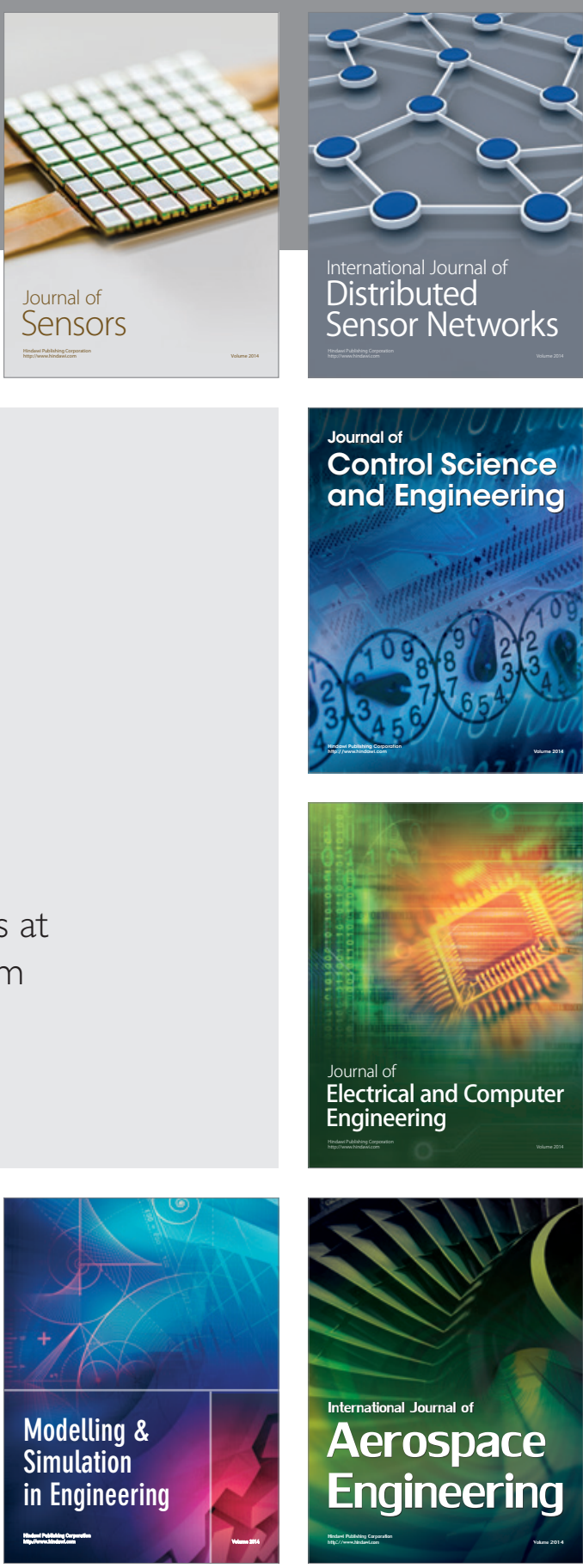

International Journal of

Distributed

Sensor Networks

Journal of

Control Science

and Engineering
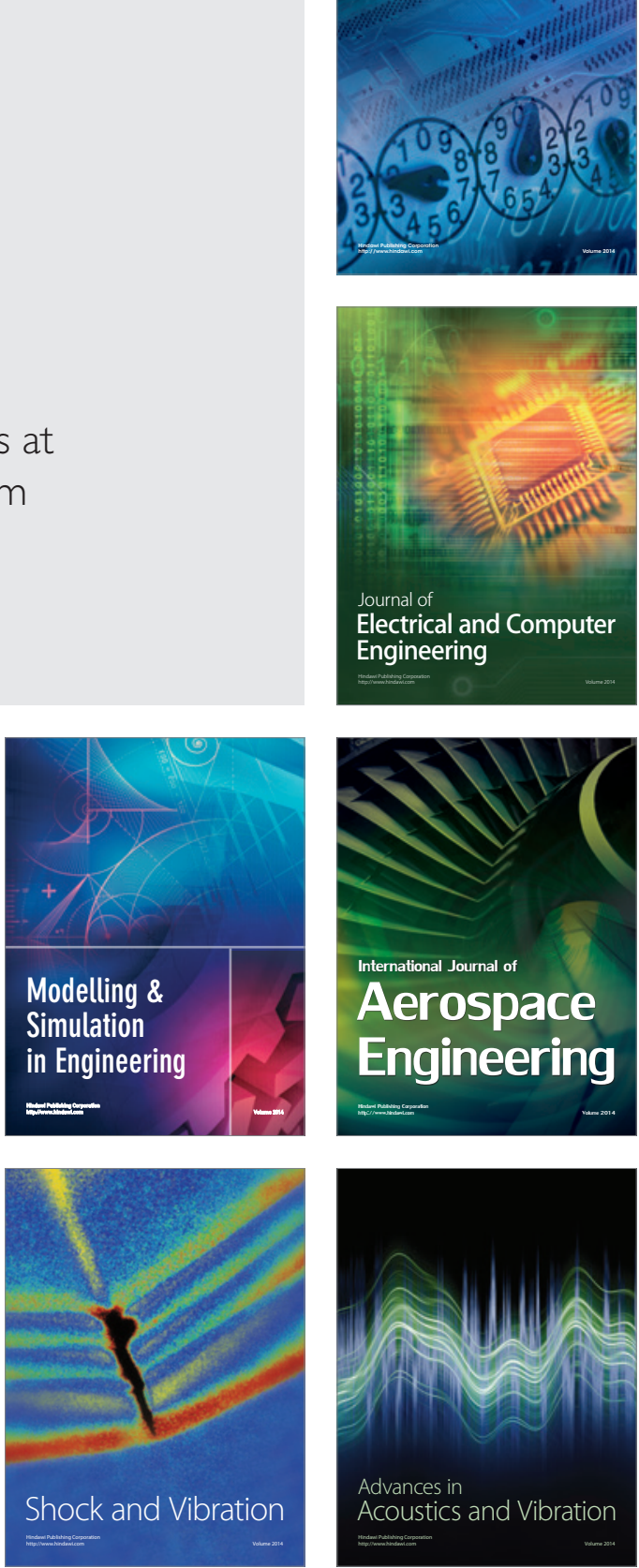